\title{
Let the Algorithm Speak: How to Use Neural Networks for Automatic Item Generation in Psychological Scale Development
}

\author{
Friedrich M. Götz ${ }^{*}$, (D) Rakoen Maertens ${ }^{2 *}$, Sahil Loomba ${ }^{3}$, and (D) Sander van der \\ Linden $^{2}$ \\ ${ }^{1}$ University of British Columbia, Canada \\ ${ }^{2}$ University of Cambridge, UK \\ ${ }^{3}$ Imperial College London, UK \\ * These authors contributed equally
}

\begin{abstract}
Author Note
Friedrich M. Götz, Department of Psychology, University of British Columbia, Canada (D) Rakoen Maertens, Department of Psychology, University of Cambridge, United Kingdom Sahil Loomba, Department of Mathematics, Imperial College London, London, UK Sander van der Linden, Department of Psychology, University of Cambridge, United Kingdom.
\end{abstract}

Accepted version, 13-September-2022.

This preprint may differ slightly from the final, copy-edited version of record, which has been published in Psychological Methods (https://doi.org/10.1037/met0000540)

This work was financially supported by the United Kingdom Economic and Social Research Council (ESRC), the Cambridge Trust and the German Academic Exchange Service $(D A A D)$. Parts of the current article are based on a conference talk presented by the second-named author at the 2021 annual convention of the Society for Personality and Social Psychology (SPSP). The authors would like to thank Dr Mark Schaller (University of British 
Columbia) for inspiring walks and thoughtful comments on the PIG and Nick Millington (APA Publishing) for flexibly granting deadline extensions as we were working through the Omicron wave of Covid-19 and the revision of the current paper. OSF repository: (https://osf.io/fhpv6/?view_only=c7d15f8becaf4ee3b8b7e59aba4de325; blinded for review) Correspondence concerning this article should be addressed to Friedrich M. Götz, Department of Psychology, University of British Columbia, 2136 West Mall, Vancouver, BC V6T 1Z4, British Columbia, Canada. Email: friedrich.goetz@ubc.ca. 


\begin{abstract}
Measurement is at the heart of scientific research. As many—perhaps most—psychological constructs cannot be directly observed, there is a steady demand for reliable self-report scales to assess latent constructs. However, scale development is a tedious process that requires researchers to produce good items in large quantities. In this tutorial, we introduce, explain, and apply the Psychometric Item Generator (PIG), an open-source, free-to-use, self-sufficient natural language processing algorithm that produces large-scale, human-like, customised text output within a few mouse clicks. The PIG is based on the GPT-2, a powerful generative language model, and runs on Google Colaboratory — an interactive virtual notebook environment that executes code on state-of-the-art virtual machines at no cost. Across two demonstrations and a pre-registered five-pronged empirical validation with two Canadian samples $\left(N_{\text {Sample1 }}=501, N_{\text {Sample2 }}=773\right)$, we show that the PIG is equally well-suited to generate large pools of face-valid items for novel constructs (i.e., wanderlust) and create parsimonious short scales of existing constructs (i.e., Big Five personality traits) that yield strong performances when tested in the wild and benchmarked against current gold standards for assessment. The PIG does not require any prior coding skills or access to computational resources and can easily be tailored to any desired context by simply switching out short linguistic prompts in a single line of code. In short, we present an effective, novel machine learning solution to an old psychological challenge. As such, the PIG will not require you to learn a new language_-but instead, speak yours.
\end{abstract}




\section{Translational Abstract}

Any research can only be as accurate as the measurement that it is based on. As many of the constructs that psychologists are interested in — such as attitudes, emotions, or personality traits - cannot be directly observed, the most common assessment method in psychological science are self-report questionnaires. However, designing good self-report measures requires the generation of large numbers of questions - also known as items - that jointly capture the psychological construct at hand. Because the demands placed on these items are high, coming up with them can take a lot of time, effort, and creativity. Moreover, the process by which good items are written remains highly elusive. Here, we propose a new solution to this old and ongoing challenge: the Psychometric Item Generator (PIG). The PIG is powered by a new generation of machine learning algorithms to produce large amounts of human-like, customised text output within a few mouse clicks. We offer a step-by-step guide, accompanied by annotated Python code and an integrated web environment to empower readers to understand, use, and adapt the PIG to suit their specific needs. We then present two applied demonstrations to illustrate the PIG's utility for the development of psychological measures. First, we show that the PIG readily generates large sets of content-relevant items for new, unstudied constructs (i.e., wanderlust). Second, we show that the PIG is able to create efficient short scales of established constructs (i.e., Big Five personality traits) that perform as well as current assessment gold standards when tested in the wild.

Keywords: automatic item generation, psychometric item generator, scale development, psychometrics, artificial intelligence, neural networks 


\section{Let the Algorithm Speak: How to Use Neural Networks for Automatic Item Generation in Psychological Scale Development}

Psychological science can only be as good as the measures that it uses. From social and personality psychology (Simms, 2008) to organizational behaviour (Hinkin, 1995; Zickar, 2020), personnel selection (Arendasy \& Sommer, 2012), and clinical assessment (Rosellini \& Brown, 2021) — measurement of psychological constructs is a fundamental element of virtually all basic and applied work in psychology (Morgado et al., 2017). Sound measurement is critical to ensure the credibility of scientific insights and guide important real-world decisions such as clinical diagnoses or the eligibility for social services (Clark \& Watson, 2019; Rosellini \& Brown, 2021).

In psychology, by far the most common form of assessment is the self-report method (Simms, 2008). Despite criticisms and calls for a greater emphasis on behavioural measures (e.g., Baumeister et al., 2007; Rauthmann, 2020), the persistent popularity of self-report measures is easy to understand: they are flexible and efficient, can be administered anywhere, to a large number of people at once and at little cost. Moreover, given the nature of psychology and its subject of study, self-reports may often be the most direct method to gather information about a construct of interest, such as people's attitudes, thoughts, emotions, or personality traits (Flake et al., 2017; Ghiselli et al., 1981; Simms, 2008). Given the enormous breadth and depth of psychological research and the virtually limitless number of psychological constructs that can be studied, it is thus no surprise that scale development is a prolific and prosperous branch of psychology — and has been for decades (Clark \& Watson, 1995, 2019). Indeed, tens of thousands of psychological scales exist, and many new ones are developed each year. To illustrate, according to a search on PsycTESTS - the American Psychological Association's official database of psychological measures, which hosts over 69,000 records - more than 337 original scales have been published in the first eight months 
of 2022 alone. At least 166 coronavirus disease 2019 (COVID-19)-related self-report scales have been developed since the global outbreak of the coronavirus in 2020, not counting translations, adaptations, or revisions of existing scales. Yet, despite its flourishing, sound scale development remains an arduous and time-consuming enterprise.

One of its most foundational and most crucial stages — and the focus of the current article - is item generation. Any new scale can only be as good as the items it is composed of, and any serious flaws with the item pool cannot be rectified at a later stage of the scale development (Clark \& Watson, 1995, 2019; Simms, 2008; Worthington \& Whittaker, 2006). Moreover, scale developers not only need to write good items, they also need to write many of them. Experts strongly advise to err on the side of over-inclusiveness when generating the initial item pool (Clark \& Watson, 2019; Loevinger, 1957), with recommendations varying from creating at least twice (Boateng et al., 2018; Nunnally, 1978), to three-to-four (DeVellis, 2016), and even five times (Schinka et al., 2012) as many initial items as one aims to include in the final scale. Over the years, many scholars have published item construction guidelines in an effort to define basic principles that facilitate the creation of good items. Amongst others, experts recommend using simple, unambiguous language appropriate for the target population, and short items that neither all nor no participants will endorse. Experts also caution against the use of jargon, slang, colloquialisms, and double-barrelled items which assess more than one characteristic (Boateng et al., 2018; Clark \& Watson, 1995, 2019; Comrey, 1988; Haladyna \& Rodriguez, 2013; Morgado et al., 2017; Rosellini \& Brown, 2021; Simms, 2008; Worthington \& Whittaker, 2006; Zickar, 2020). Nonetheless, there is no simple formula to write a perfect item (Clark \& Watson, 1995; DeVellis, 2016; Rosellini \& Brown, 2021), and in the eyes of many, item generation remains an art (Gierl et al., 2012; Zickar, 2020) that requires substantial time, effort, consideration, and creativity. 
The combination of a continuous demand for good items in psychological science and the difficulty of producing them raises the question about more cost-effective ways to generate items. In the current paper, we first describe and review the basics of existing approaches to automated item generation, statistical language modelling, and modern natural language generation methods. In so doing, we argue that openly accessible state-of-the-art AI-driven language models offer a fast, scalable, and versatile alternative to traditional manual item generation. We then go on to demonstrate through a hands-on tutorial that this new approach requires minimal technical understanding and no personal computing resources and produces output comparable to human-generated items. Finally, we carry out a fivepronged psychometric validation study to provide real-world empirical evidence that our unedited, algorithmically-generated items not only form cogent, reliable, and valid scales but that these scales can compete with the performance of traditionally-developed gold standard measures.

\section{Automated Item Generation (AIG)}

The idea of automated item generation (AIG) — using computer algorithms to produce items (Embretson \& Yang, 2007; Gierl \& Haladyna, 2012; Irvine \& Kyllonen, 2002)—is not entirely novel. In fact, neighbouring disciplines have been using AIG for some time. Perhaps the most prominent application so far has been testing in medical education. Here, AIG has been successfully employed to produce multiple-choice questions that do not only yield desirable psychometric properties but are also virtually indistinguishable from manually generated items when rated by content specialists (Gierl \& Lai, 2013; Gierl et al., 2016; Pugh et al., 2020). Similarly, AIG has been used for the creation of diverse mathematical test items (Arendasy \& Sommer, 2012; Kosh et al., 2019), including figural matrices (Arendasy \& Sommer, 2005), mental rotation items (Arendasy \& Sommer, 2010), and algebra word problems (Arendasy \& Sommer, 2007), as well as to generate short music snippets for 
melodic discrimination testing (Harrison et al., 2017). What unites these successful previous applications is that they 1) all produced test items (with correct and false answers) and 2) relied on generating clones of narrowly defined item types by varying singular parameters (von Davier, 2018). To generate these types of items, researchers typically follow a threestage process (Gierl et al., 2012): first, content experts develop a cognitive model (i.e., a theoretical model of how items will be processed and solved); second, content experts create an item model (i.e., a prototypical representation of the desired item type); third and lastbased on the previous steps - a computer algorithm uses different combinations of content elements to generate new items. While this approach has been very helpful in creating test items that follow a pre-determined structure and have a finite set of contents that can be freely combined, it is easy to see that it might be less helpful in generating Likert-type items that assess attitudes or personality traits (von Davier, 2018). The aforementioned process also highlights other significant start-up costs that come with AIG: first, in the form described above, AIG is anything but automatic and extensive preparatory steps are required before any automation takes place (Kosh et al., 2019). Second, in order to actually use computer algorithms, one must either be able to train them — which requires expertise and large computational storing and processing capacities (Fetaya et al., 2020) —or gain access to existing software, which has hitherto not been widely available (Royal et al., 2018).

In the following sections, we showcase a solution which—in our view-removes each of these entry barriers and offers a flexible platform for automated large-scale generation of items suited for diverse applications in psychological science. In so doing, we leverage a powerful natural-language-processing machine learning model, the Generative Pre-Trained Transformer 2 (GPT-2; Radford et al., 2019b) that uses deep neural networks to generate coherent, realistic, grammatically correct text. To that end, we first provide a brief overview 
of the basics of statistical language modelling and recent advancements in natural language processing (NLP).

\section{Statistical Language Modelling}

At the nexus of linguistics and machine learning, natural language processing (NLP) is a burgeoning and fast-growing field that has its conceptual and computational roots in statistical language models. In essence, statistical language models employ functions (or algorithms designed to learn such functions) to build probabilistic representations of natural language that reflect as realistically as possible the order and combinations in which specific words may be used in text. Based on these representations, language models can then make probabilistic predictions of which words can follow a given string of words (also referred to as tokens, Manning et al., 2008). A sequence of ground-breaking methodological innovations in recent years has enabled massive advances in NLP and made language models more efficient, realistic, and powerful. The first such recent event ushering in significant change was the introduction of neural networks in language modelling (Bengio et al., 2003). Previous statistical language models had largely been reliant on the existing number of occurrences of words, pairs of words, and higher-order word combinations to mine linguistic patterns, and thus quickly ran into the problem of combinatorial explosion, unable to generalize to new word combinations. Neural language models parametrise the statistical model by learning continuous representations of words given their embedding within a wider context, allowing words appearing in similar contexts to "borrow strength" from one another, thus generalizing to entirely unseen word combinations. They are able to automatically organize concepts and learn rich language structures from textual data (Han et al., 2021; for an excellent book on neural network methods in NLP see Goldberg, 2017). For instance, they can implicitly learn the relationship between countries and their capital cities: what "Berlin" is to "Germany", "Paris" is to "France" (Mikolov et al., 2013). To do so, neural networks 
exploit the capability of a non-polynomial transformation of linearly combined inputswords in a given context - to approximate an arbitrary function of those inputs (Hornik et al., 1989) - the probability of occurrence of that word in the given context. Each computational unit, termed a "neuron", performs a single such transformation, and iterative application of such transformations by "stacking" multiple layers of neurons in a "deep" neural network architecture permits learning richer functions more efficiently (Telgarsky, 2016). Thanks to this, deep neural networks — particularly recurrent neural networks (RNNs; Elman, 1990; Mikolov et al., 2010; Liu et al., 2016; Sutskever et al., 2014; Wu et al., 2016) that include recurrent connections between layers letting the network retain information from more distant contexts-learn richer and more contextualized word and phrase relationships, and have achieved tremendous success and popularity in NLP. The addition of "attention" mechanisms allows a word to automatically attend to selective parts of a sentence, including those further away, instead of fixed-length context windows (Bahdanau et al., 2016). However, these deep networks suffer from two key weaknesses that impede performance and efficiency. First, they struggle with very long-range dependencies, which prevents them from attaining human-like performance. That is, as sentences get longer and words are spread further apart from each other, the likelihood of using context from a word at the beginning of the sentence for the prediction of a new word at the end of the sentence decreases exponentially (Linzen \& Baroni, 2021; Topal et al., 2021; Yang, 2021). Second, due to recurrent connections, RNNs must process input sentences sequentially, one word at a time. That is, they are unable to carry out any computational step until they have completed the previous step, which precludes parallelisation and hence the possibility to learn the model on very large texts (Linzen \& Baroni, 2021; Topal et al., 2021; Yang, 2021). The next incisive development within NLP that sought to overcome these limitations was the advent of the transformer architecture (Vaswani et al., 2017). The transformer is a very deep neural network that 
ditches the recurrent connections but retains the attention mechanism. In practice, this means that transformers can relate every word in an input sequence to every other word, which makes transformers equally good at computing long-range (i.e., between words that are far apart) and short-range (i.e., between words that are proximate) dependencies (Lake \& Murphy, 2022; Launay et al., 2021; Vaswani et al., 2017; Wolf et al., 2020). Lack of recurrence implies that transformers can process all words in a given sentence in parallel, rather than sequentially, which enables parallelisation and greatly speeds up computation times (Launay et al., 2021; Vaswani et al., 2017; Wolf et al., 2020). As a result, much larger, deeper, and more expressive models can be trained that accomplish richer contextual embeddings for individual words - allowing them, amongst others, to better differentiate between homographs (i.e., words with multiple meanings such as "arms", "bank", or "sex")—and offer a more complex and accurate general representation of language (Han et al., 2021; Launay et al., 2021).

In combination with the rapidly rising availability of high-quality large-scale text corpora, and hardware capabilities to learn models with millions of parameters, these advances have led to the recent development of various powerful transformer-based statistical language models (e.g., BART, Lewis et al., 2019; BERT, Devlin et al., 2018; GPT-2, Radford et al., 2019b; T5, Raffel et al., 2019; XLNET, Yang et al., 2019) that perform diverse tasks: from language understanding, classification, word insertion, and question answering, to summarisation, translation, and essay writing (Han et al., 2021).

\section{The GPT-2}

While these new-generation language models perform best in different tasks depending on the purpose for which they were trained and developed, given our goal of automated item generation for psychological assessment, in this tutorial we will focus on one specific model that has been explicitly designed for open-ended text generation: the 
Generative Pre-Trained Transformer-2 (GPT-2; Radford et al., 2019b). The GPT-2 is, as the name suggests, a neural language model based on the transformer architecture (Vaswani et al., 2017). The GPT-2 has been developed by OpenAI (Radford et al., 2019b), an AI research and deployment company, and is freely available to researchers. Harnessing the massive scalability afforded by the transformer architecture, its full version features 1.5 billion parameters that have been trained on a dataset of eight million web pages, worth $40 \mathrm{~GB}$ of text, to learn the conditional probabilities of words given previous words within a text. Once "trained", words can be "sampled" from this statistical model to generate new text that is likely to follow any conditioning text, without requiring any further fine-tuning, making it a "generative pre-trained" model. The model is autoregressive, which means that it uses all previous words in a sequence, including those it has generated itself during prior generation steps, as context to predict and generate subsequent words. The generated text tends to be coherent, grammatically correct, and shows a chameleon-like adaptation to the content and style of the conditioning text, making it an extremely flexible tool that can be used to generate realistic continuations on any written prompt. Attesting to its power and unprecedented ability to generate human-like text, the GPT-2 has been used to create an open-ended multiplayer text adventure game (Risi \& Togelius, 2020; AI Dungeon https://play.aidungeon.io/main/landing), write poetry (https://www.gwern.net/GPT-2; see also: Köbis \& Mossink, 2021), generate patent applications (Lee \& Hsiang, 2019) and come up with an opening speech in a debating competition that was judged to be almost as convincing as its human-written counterpart (Slonim et al., 2021). In the remainder of the current paper, we describe and demonstrate a GPT-2-based application — the Psychometric Item Generator (PIG) - that we have set up to enable researchers to generate unlimited amounts of customised items in a few simple steps without any prior coding knowledge or access to high-performance computing facilities. For a detailed account of the training and 
application of statistical language models, as well as a technical description of their underlying mathematical equations, we refer the reader to Supplement S1 in the Supplementary Information.

\section{Using the GPT-2 for AIG: An Introduction to the Psychometric Item Generator (PIG)}

Our GPT-2 application for automatic item generation, the Psychometric Item Generator (PIG), is hosted on Google Colaboratory ${ }^{1}$. Google Colaboratory-Colab for short - is a free cloud-computing platform that provides an interactive serverless notebook environment in which multiple users can write, share, and execute code (Bisong, 2019). Colab operates on Jupypter notebooks, which are compatible with a number of leading programming languages, including Python, $\mathrm{R}$ and Julia, and allow researchers to intermix narrative explanations, software code, computational output, and multimedia resources in a single workflow document (Perkel, 2018). To run Colab notebooks, Google grants access to Linux-based remote virtual machines featuring state-of-the-art hardware with advanced graphics processing units (GPUs; e.g., K80 or T4 Nvidia) for runtimes of up to 12 hours (Nelson \& Hoover, 2020). Colab notebooks are customarily stored on Google Drive but can also be saved to GitHub or downloaded to a local machine (Bisong, 2019). As a fully cloudbased platform, Colab does not require any installation and can be directly accessed via a web browser. In the following, we describe how to access and operate the PIG on Colab in an annotated step-by-step guide.

1. Access the PIG via our project website (misinfo.uk/AIG) or directly under https://colab.research.google.com/drive/1ZR32-zBm16-ZGUSzSefSC49fb7LMobgf. ${ }^{2}$

Upon opening the Colab notebook you should see five blocks of annotated Python code. For each block, annotations in green precede Python commands in black and

\footnotetext{
${ }^{1}$ Creation and implementation of the PIG have been inspired and greatly facilitated by various excellent AI blogs and open-source code-sharing resources, including Woolf (2019), Nasser (2020) and van Platen (2020). 2 The Python scripts can also be found on the OSF repository for this paper: https://osf.io/fhpv6/?view only=c7d15f8becaf4ee3b8b7e59aba4de325 (blinded for review).
} 
blue. Once a command has been carried out, a short summary note will pop up at the bottom of your browser window, notifying you how long it took to run the respective step and at what time the process was completed. In addition, any output will appear right below the block in question.

2. Run the first block by pressing "Shift+Enter" or hitting the "play" button on its left.

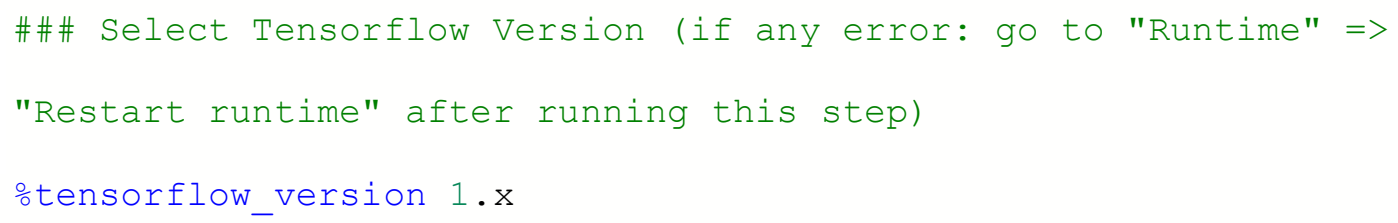

Running this first block loads Tensorflow 1.x. Tensorflow is an open-source software library for developing and training machine learning models with a particular focus on deep neural networks (Abadi et al., 2016; for a book-length introduction to NLP using Tensorflow see Ganegedara, 2018). We use Tensorflow 1 to ensure backward compatibility and endured functioning of the PIG long after the publication of this article. ${ }^{3}$ Upon successfully executing this block a message reading "TensorFlow 1.x selected" will appear right below the code. If you receive any error messages when running this line of code, please go to "Runtime"- that is the fourth column in your menu at the top of the page — and select "Restart runtime".

3. Run the second block.

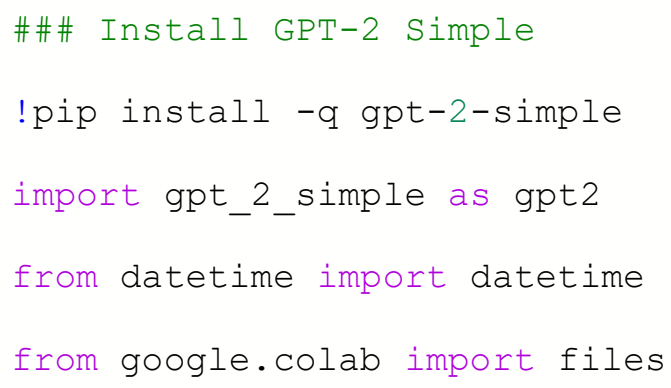

\footnotetext{
${ }^{3}$ Newer versions of Tensorflow may work but could require some additional coding to set up.
} 
Here, the notebook installs the GPT-2 and its dependencies and loads the package. In so doing, it uses a wrapper function (Woolf, 2019), that hides the real complexity of the GPT-2.

4. Run the third block.

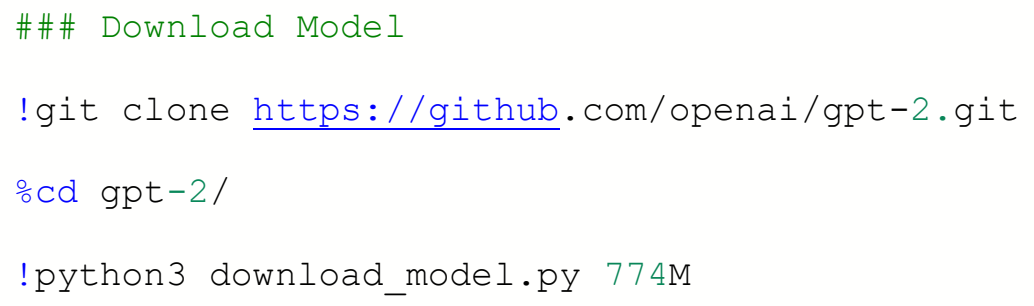

This code first clones the GPT-2 repository from the OpenAI GitHub page and then downloads the pre-trained GPT-2 model from the Google Cloud storage and saves it in the Colab virtual machine. Four models are available (Solaiman et al., 2019): small (124M hyperparameters), medium (355M hyperparameters), large (774M hyperparameters) and full (1558M hyperparameters). As the models get larger, they require greater computational resources and more disk space and take longer to generate output but also produce better results as the models have more knowledge to draw from. Here, we use the large model (774M hyperparameters), which takes up 3.1 GB of disk space. If you wish to choose any of the other models, you can do so by simply adjusting the last line of code in the current block (e.g., from "!python3 download_model.py 774M” to “!python3 download_model.py 355M”). Importantly, at the time of writing, the larger models $(774 \mathrm{M}, 1558 \mathrm{M})$ cannot currently be finetuned on Colab, although they can be used to generate text from the pre-trained model, which is how they will be used here. Moreover, the full $1558 \mathrm{M}$ model requires very fast GPU support and may not work, depending on which resources are attached to the Colab notebook (Woolf, 2019). We therefore recommend using the 774M model for smoothest overall experience and best effort-output ratio. Lastly, please note that 
whichever model you choose, you are downloading an extremely large model and the process may thus take some time.

5. Run the fourth block.

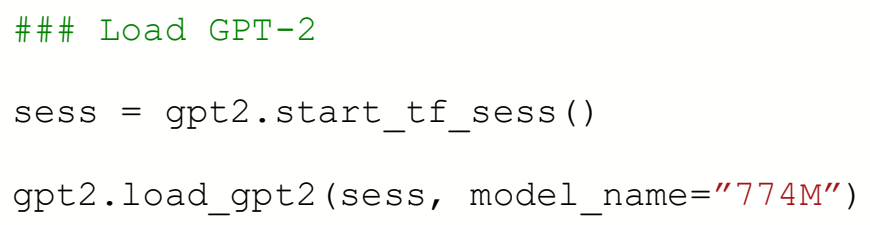

This block starts a new Tensorflow session, wherein it loads the pre-trained 774M GPT-2 model which we will use to generate text in the sixth and last step.

6. Run the sixth block.

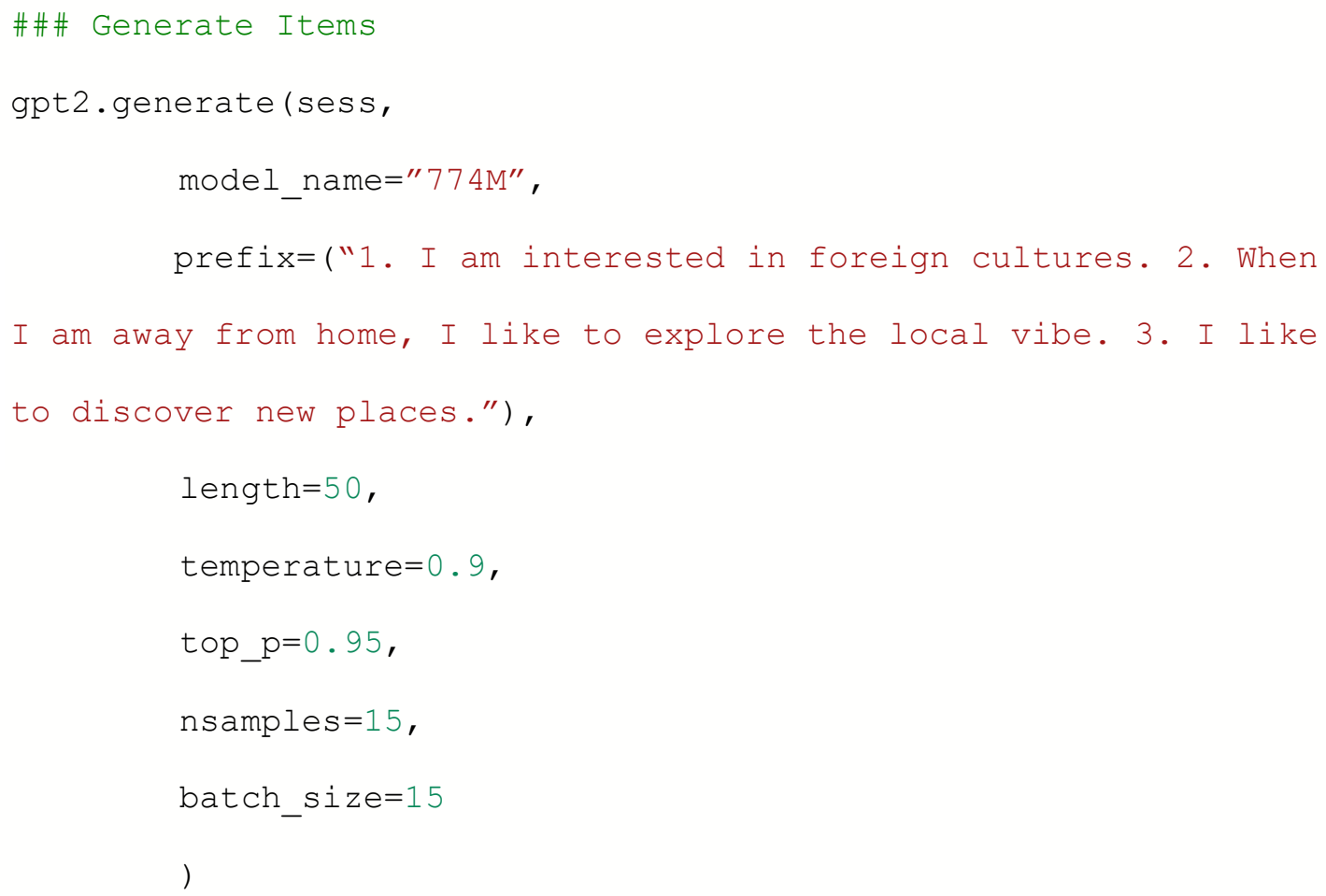

Here we leverage the pre-trained 774M GPT-2 language model to generate new items for psychological scale development based on as little input as a single sentence. As this block offers various opportunities for customised adaptation and individual tweaking, we explain its components in more detail below. This command generates 
items by executing a Tensorflow session (sess), that is based on the 774M GPT-2 model (model_name $=$ "774M") loaded in Step 5. Prefix indicates the beginning of the text that will then be automatically continued. This is where we insert our input. For example, if you are planning to create a new wanderlust scale, you might write: "prefix=("I am interested in foreign cultures. "),". For better results, we recommend using multiple sentences, such as "prefix $=($ "1. I am interested in foreign cultures. 2. When I am away from home, I like to explore the local vibe. 3. I love to discover new places."),". ${ }^{4}$ The next parameters enable users to configure and fine-tune their output. Length refers to the number of tokens - that is words within a text - to be generated. Our default setting for the PIG is 50. Temperature and top_p regard the creativity of the output text. Remember that the GPT-2 has been trained to predict the most probable next word given all existing words within a text. If the algorithm always chose the single most likely word, the generated text would quickly get very repetitive, and the algorithm would restrict itself to using a few high-probability words multiple times. Parameters such as temperature and top_p are designed to manipulate the creativity of the output by varying the randomness with which the algorithm selects the next word. To do this, temperature adjusts the sharpness of the conditional next word distribution (Ackley et al. 1985). That is, the lower the temperature, the higher the likelihood for the algorithm to select high-probability words and the lower the likelihood to select low-probability words, which means decreased randomness, variation, and creativity in the generated output. At the extreme of temperature $=0$, this equals so-called greedy decoding - that is, the

\footnotetext{
${ }^{4}$ To make the code easier to read, a line break can be introduced by including Python's line break symbol "In": prefix $=(" 1$. I am interested in foreign cultures. $\mid n "$

"2. When I am away from home, I like to explore the local vibe. In"

"3. I love to discover new places. $(n$ "),
} 
algorithm will always and without exception select the most probable word to continue the text. On the flip side, setting temperature at very high levels might produce diverse and creative yet chaotic, unintelligible gibberish. Following current best practice, we therefore recommend keeping temperature between 0.7 and 1.0 (Woolf, 2019) and choose 0.9 as our default (Holtzmann et al., 2020; Nasser, 2020)_ which allows the model to make "suboptimal" predictions for the sake of linguistic diversity, without jeopardising the coherence of the resulting text. In a similar vein, top- $p$ or nucleus sampling (Holtzmann et al., 2020) provides an arguably even more elegant way to harness probabilistic word selection to improve linguistic diversity. Instead of always sampling the next word from a set number of the $k$ most likely words (so-called top- $k$ sampling; Fan et al., 2018), top_p sampling selects the next word from the so-called nucleus, that is, the smallest possible set of words whose cumulative probability exceeds the pre-determined probability $p$. This means that 1 ) the higher one sets $p$, the more words will enter the nucleus that the algorithm picks from, and 2) the size of the nucleus changes dynamically, depending on the last known word. For example, if the last word is "the", there are lots of well-fitting options for the next word, which makes the probability for each individual word smaller and in turn means that the nucleus (i.e., the set of words whose cumulative probability exceeds $95 \%$ - assuming we set $p$ to 0.95 ) will be larger. In contrast, if the last word is "tsunami", there are only relatively few well-fitting options for the next word, which makes the probability for each individual word bigger and in turns means that the nucleus will be smaller. Thus, the size of the nucleus dynamically contracts or expands as a function of the last word and the threshold of $p$. The higher $p$, the larger the nucleus, the more words are available for the algorithm to choose from and thus the more diverse the resulting text. Following prior research, we set 
top_p to 0.95 (Holtzmann et al., 2020). Summed up, temperature and top_p are restricted sampling approaches that foster linguistic diversity while truncating the low-confidence tail of the probability distribution (i.e., ruling out the vast majority of unlikely words in favour of a small dynamic set to choose from) in order to produce more diverse and human-like text without threatening the fluency and readability of the output (Holtzmann et al., 2020). More details on sampling strategies, including corresponding mathematical equations, are provided in Supplement S1. The last two parameters, nsamples and batch_size, are relevant for automated bulk generation, which is one of the big advantages that the PIG holds over manual item writing. Herein, nsamples refers to the total number of text outputs to be generated while batch_size describes the number of text outputs generated at a time. At present, Colab GPUs support a maximum batch_size of 20 (Woolf, 2019). Hence, with our recommended settings $($ nsamples $=100$, batch_size $=20)$ you can generate 100 text output samples in less than one minute.

Having thus explained the general set-up and inner workings of the PIG, we will now illustrate its utility for psychological scale construction across two contexts designed to mimic the predominant classes of scale building endeavours (i.e., creating measures of new constructs and creating more efficient measures of existing constructs). In Demonstration 1, we will use the PIG to generate a large pool of face-valid items for the assessment of a novel construct (i.e., wanderlust) for which there are no pre-existing measures. In Demonstration 2, we will use the PIG to create new semi-short scales for the assessment of established constructs (i.e., the Big Five personality traits) and formally evaluate their performance vis-àvis the original measure. 


\section{Psychometric Item Generator (PIG) Demonstration 1: Creating a Large Pool of}

\section{Wanderlust Items}

Let us imagine we wanted to develop a measure of "wanderlust" based on a conceptualisation of wanderlust as a deep-seated longing for discovery and exploration of, as well as immersion in foreign cultures and places. Based on this conceptual starting point, we write down three initial items that we will feed into the PIG to kick off our large-scale automated item generation process. These items are: "1. I am interested in foreign cultures.", "2. When I am away from home, I like to explore the local vibe.", and "3. I love to discover new places.". Next, we enter these items into our language model by inserting them in the prefix section of our gpt2.generate-command in the last code block. We do not make any other changes and simply execute the Colab notebook. Results from a single execution-with a runtime of 55 seconds-yielded 65 items that pass an initial face validity check and could be added to a larger item pool for the development of a wanderlust scale. ${ }^{5} \mathrm{We}$ list these cherry-picked unedited items in Table 1 and provide the entire text output in the online materials (OM1), hosted on our Open Science Framework project page (https://osf.io/fhpv6/?view only=c7d15f8becaf4ee3b8b7e59aba4de325; blinded for review)

\section{Table 1}

Selected Wanderlust Scale Candidate Items from a Single Iteration of the PIG

1. I enjoy spending time in different cultures.

2. I enjoy hearing different views and different languages.

3. I am always open to new experiences and new people.

4. I am an internationalist.

5. I love places that are interesting and that you can walk around and enjoy the atmosphere.

6. I am interested in learning more about the local cultures.

7. I am curious about languages.

8. I like to find an unusual place to travel.

9. I find the process of walking around interesting.

10. I am the kind of person who likes to go with other people on a journey.

11. I am drawn to people with different backgrounds and experience.

\footnotetext{
${ }^{5}$ All output that we report stems from the first execution of our code.
} 
12. I enjoy traveling the world.

13. I am always happy to enjoy new experiences.

14. I love to travel. (x3)

15. I got that the best way to understand a culture is to try it.

16. I like exploring new places, different cultures, things I have never even heard of, and so forth.

17. I want to travel and explore different cultures.

18. If I have the opportunity, I would like to travel abroad to speak to people from all over the world.

19. I like to travel very often.

20. I enjoy traveling.

21. I like to communicate with people around the world.

22. I am a "true traveler."

23. I would like to learn more about the local culture.

24. I am curious about how different cultures live and work.

25. I like to go on long walks, exploring nature, the countryside, and any other places where I feel the grass is always greener.

26. I enjoy traveling and exploring.

27. I am a free spirit.

28. I do not like restrictions on my imagination and creativity.

29. I like to use my imagination to explore and make a living.

30. I am proud to have traveled the world.

31. I am not afraid to be different or go against the grain.

32. I want to learn about myself and the world around me.

33. I want to explore, both in my own country and abroad.

34. I am a romantic traveler.

35. I enjoy new foods.

36. I enjoy travelling and meeting new people.

37. I am generally an extremely open-minded person who is more receptive to ideas from other cultures.

38. I am willing to leave my comfort zone and travel.

39. I am interested in discovering the newest Indian foods.

40. I can talk to strangers.

41. I like to take a walk on the wild side.

42. I like to travel abroad.

43. When I am in the town, I like to hang out in local restaurants, bars, and stores.

44. I believe in frequent travel and adventure.

45. I love exploring new local foods.

46. I enjoy traveling, particularly to new areas.

47. I am an outdoorsman.

48. I like to move to new cities.

49. I often spend weekends away from home. 
50. I enjoy international travel.

51. I like to see what cultures and languages they speak.

52. I have an affection for travelling.

53. I am an adventurer and adventure seeker.

54. I have a great interest in the outdoors.

55. When traveling, I seek out social experiences.

56. I want to take pictures of my surroundings, stories and places.

57. I am always on the move.

58. I enjoy meeting people and have always wanted to travel more.

59. My favorite activity is outdoor exploring.

60. I prefer to read books and magazines about foreign cultures.

61 . I want to try a new type of cuisine.

62. I love traveling and living abroad.

63. I like to study the history and culture of a particular place.

64 . I would like to learn a new language.

65. I am active and adventurous.

We believe that these results aptly highlight various benefits of the PIG. First, scalability - it took less than one minute to generate over 60 passable wanderlust items, and we can repeat this as often as we like. Second, the PIG may not only serve well to generate large numbers of new items, but we can also think of it as an inductive, data-driven bottomup tool that can help to expand and refine our conceptual thinking on the construct that we are trying to measure. This is true in a number of ways: the deep neural networks that underlie the GPT-2 and hence the PIG may make visible associations and connections between our initial input and other behaviours, emotions, and cognitions that we did not think of when starting to develop a wanderlust scale. For example, items from our first iteration (see Table 1) featured themes such as adventure, exploring the outdoors, learning about new cuisines and languages, and living abroad. Compared to the three items that we started with the newly created items reflect a much broader, richer range of other potential markers of wanderlust. In other words, the PIG may help scale developers to free themselves from their own restrictive biases and embrace the development of a concept whose breadth and depth are no longer subject to the limitations of their own initial conceptualisation. 
Other output may not be directly useable for the creation of an initial wanderlust item pool but may offer helpful other cues. For example, the current output contained sentences such as "I value my independence", "I am outgoing and excited to meet new people", or "I'm not afraid to go through obstacles" (see OM1; https://osf.io/fhpv6/?view_only=c7d15f8becaf4ee3b8b7e59aba4de325; blinded for review), which may help us to think about the nomological network of the construct that we are seeking to develop. In this context, such items may suggest that wanderlust could be positively related to existing psychological constructs such as individualism, extraversion, and risk-taking. And last but not least, some of the output, such as "My unstructured life is the perfect vacation", can be outright entertaining. In other words, when using the PIG, item generation may not only be extremely fast and very inclusive but also fun.

\section{Psychometric Item Generator (PIG) Demonstration 2: Development and Validation of a}

\section{Big Five Personality Trait Measure}

As is evident from the demonstration above, the PIG can effortlessly generate large and diverse sets of items with a high degree of face validity. That is, these items intuitively look like they would likely be good indicators of the latent construct at hand - in this case wanderlust. However, to make a compelling case for widespread adoption of the PIG, we need to provide empirical evidence that the items generated by the PIG do in fact form fully functional scales which hold up under psychometric scrutiny and perform as well as established measures that were developed through traditional means. Thus, in this second demonstration we employed the PIG to develop a new, alternative 20 -item semi-short scale for the assessment of the Big Five personality traits (i.e., Openness, Conscientiousness, Extraversion, Agreeableness, Neuroticism or OCEAN for short; John \& Srivastava, 1999).

Subsequently, the newly developed measure, called the N-BFI-20 (short for NeuralBig Five Inventory-20) was subjected to rigorous psychometric vetting and benchmarked 
against the BFI-2 (Soto \& John, 2017), a full-length 60-item measure and the current gold standard for Big Five assessment. As this new demonstration effort involved the collection of original data from human participants, prior to carrying out the study we obtained ethical approval from the Behavioural Research Ethics Board at the University of British Columbia (application number: H21-03154). All analyses and directional hypotheses featured in this scale development and validation study were pre-registered on the Open Science Framework (https://osf.io/fhpv6/?view_only=c7d15f8becaf4ee3b8b7e59aba4de325; blinded for review). In keeping therewith, we adopted a five-step approach.

\section{STEP 1: Generation of a Large Item Pool Through the PIG}

In STEP 1, we generated a large pool of item candidates by inputting items from the existing Big Five Inventory 2 (BFI-2; Soto \& John, 2017) into the Psychometric Item Generator (PIG). In so doing, we ran 10 different input iterations. That is, for each Big Five dimension (i.e., Openness, Conscientiousness, Extraversion, Agreeableness, Neuroticism; John \& Srivastava, 1999) we ran two iterations, one including all regular-keyed items and one including all reverse-keyed items. Across all iterations the default parameter settings for the PIG were used as described above (i.e., length $=50$, temperature $=0.9$, top_p $=0.95$, nsamples $=100$, batch_size $=20)$. Table 2 lists all iterations and corresponding input items (entered into the PIG through the input section called prefix).

\section{Table 2}

PIG Input for N-BFI-20 Item Pool Generation

\section{Iteration}

Iteration 1

(Openness, regular-keyed)
Input

1. I am someone who is curious about many different things.

2. I am someone who is inventive, finds clever ways to do things.

3. I am someone who is fascinated by art, music, or literature.

4. I am someone who values art and beauty. 
Iteration 2

(Openness, reverse-keyed)

Iteration 3

(Conscientiousness, regular-keyed)

Iteration 4

(Conscientiousness, reverse-keyed)

\section{Iteration 5}

(Extraversion, regular-keyed)

Iteration 6

(Extraversion, reverse-keyed)

\section{Iteration 7}

(Agreeableness,

regular-keyed)

Iteration 8

(Agreeableness, reverse-keyed)
5. I am someone who is complex, a deep thinker.

6. I am someone who is original, comes up with new ideas.

1. I am someone who has few artistic interests.

2. I am someone who avoids intellectual, philosophical discussions.

3. I am someone who has little creativity.

4. I am someone who has difficulty imagining things.

5. I am someone who thinks poetry and plays are boring.

6. I am someone who has little interest in abstract ideas.

1. I am someone who is dependable, steady.

2. I am someone who is systematic, likes to keep things in order.

3. I am someone who keeps things neat and tidy.

4. I am someone who is efficient, gets things done.

5. I am someone who is reliable, can always be counted on.

6. I am someone who is persistent, works until the task is finished.

1. I am someone who tends to be disorganized.

2. I am someone who tends to be lazy.

3. I am someone who has difficulty getting started on tasks.

4. I am someone who can be somewhat careless.

5. I am someone who leaves a mess, doesn't clean up.

6. I am someone who sometimes behaves irresponsibly.

1. I am someone who is outgoing, sociable.

2. I am someone who has an assertive personality.

3. I am someone who is dominant, acts as a leader.

4. I am someone who is full of energy.

5. I am someone who is talkative.

6. I am someone who shows a lot of enthusiasm.

1. I am someone who rarely feels excited or eager.

2. I am someone who tends to be quiet.

3. I am someone who is less active than other people.

4. I am someone who is sometimes shy, introverted.

5. I am someone who finds it hard to influence people.

6. I am someone who prefers to have others take charge.

1. I am someone who is compassionate, has a soft heart.

2. I am someone who is respectful, treats others with respect.

3. I am someone who has a forgiving nature.

4. I am someone who is helpful and unselfish with others.

5. I am someone who is polite, courteous to others.

6. I am someone who assumes the best about people.

1. I am someone who tends to find fault with others.

2. I am someone who feels little sympathy for others.

3. I am someone who starts arguments with others. 
Iteration 9

(Neuroticism, regular-keyed)

Iteration 10

(Neuroticism, reverse-keyed)
4. I am someone who is sometimes rude to others.

5. I am someone who is suspicious of others' intentions.

6. I am someone who can be cold and uncaring.

1. I am someone who is moody, has up and down mood swings.

2. I am someone who can be tense.

3. I am someone who worries a lot.

4. I am someone who often feels sad.

5. I am someone who tends to feel depressed, blue.

6. I am someone who is temperamental, gets emotional easily.

1. I am someone who is relaxed, handles stress well.

2. I am someone who stays optimistic after experiencing a setback.

3. I am someone who feels secure, comfortable with self.

4. I am someone who is emotionally stable, not easily upset.

5. I am someone who keeps their emotions under control.

6. I am someone who rarely feels anxious or afraid.

For each iteration the PIG generated 100 sets of output (as specified by setting nsamples to 100). With the rare exception of single output sets in which the PIG deviated from the inputted sample item structure, almost all output sets yielded seven to 10 sentences that conformed to the desired item format. Of these, the first six are always a repetition of the input (the conditioning text), leaving one to four new items per output set. Thus, each of the 10 iterations generated about 200 to 300 item candidates, which formed the basis for preselection of an initial high-quality item pool through our double-blind expert committee in STEP 2. The complete output for each iteration is exhibited in OM2 in the online materials (https://osf.io/fhpv6/?view only=c7d15f8becaf4ee3b8b7e59aba4de325; blinded for review).

\section{STEP 2: Pre-selection Through Double-Blind Expert Committee}

In STEP 2, we aimed to condense the large number of item candidates generated in STEP 1 into a smaller number of promising item candidates which would subsequently be subjected to empirical testing to extract the final measure (i.e., the Neural-BFI-20; see STEP 
3). To accomplish this goal, we followed prior research (Kohno et al., 2021; Schueler et al., 2014; Zielinski \& Veilleux, 2018) and recommended practices in psychometrics (Carpenter, 2018; Gehlbach \& Brinksworth, 2011; Haynes et al., 1995; Simms, 2008) and set up a double-blind expert committee. Said committee consisted of four researchers from four different cultural backgrounds (Belgium, Germany, India, the Netherlands), all of whom have a clear understanding of personality psychology, the Big Five and psychometric scale development. Each expert independently reviewed all 1,000 output sets (100 per iteration) generated in STEP 1. To guide item selection in a principled way, experts were instructed to base their decision on conceptual relevance and linguistic clarity (Gehlbach \& Brinksworth, 2011; Schueler et al., 2014), and flag up every item that they believed might be a viable item and would not require any language editing. In addition, in making their selections experts were encouraged to remove redundant items as well as items that were overly similar to the source items from the BFI-2. As laid out in our pre-registration (https://osf.io/fhpv6/?view only=c7d15f8becaf4ee3b8b7e59aba4de325; blinded for review), we initially proposed that all items that achieved at least a three-out-of-four-consensus would be considered for the next stage (i.e., STEP 3: empirical testing). As a boundary condition, we had determined that at a minimum, we aimed to retain eight items per personality dimension — and within those four regular-keyed and four reverse-keyed items, amounting to a total of at least 40 items (i.e., at least twice as many as the final measure; Boateng et al., 2018; Nunnally, 1978). Upon inspection of the independent expert selections, it became evident that adopting a three-out-of-four-consensus as inclusion threshold would result in an unnecessarily large item pool. Faced with the luxurious problem of having far too many candidate items that were deemed to be of sufficient quality by at least three experts, we thus decided to impose an even more stringent decision rule and opted to retain only items that had gathered unanimous support (i.e., four-out-of-four consensus). This more stringent 
criterion was met by at least six items from nine of the 10 iterations. For the third iteration (Conscientiousness, regular-keyed), only three items met the four-out-of-four-consensus and we thus relaxed our rule to include another three items that achieved a three-out-of-four consensus for this iteration. As a result, overall, 92 unedited items were retained, providing firm support for the utility of the PIG and forming a rich and well-balanced N-BFI item pool for empirical testing in STEP 3. The final item selections for each iteration are displayed in Table 3.

Table 3

N-BFI Item Pool Based on Double-Blind Expert Committee Selections

Iteration Item Selections

Iteration 1

(Openness, regular-keyed)

Iteration 2

(Openness, reverse-keyed)
1. I am someone who is interested in new ideas.

2. I am someone who is able to think outside the box.

3. I am someone who is curious, willing to try anything.

4. I am someone who cares about the arts.

5. I am someone who likes learning new things.

6. I am someone who is always seeking information.

7. I am someone who is full of ideas.

8. I am someone who thinks outside the box.

9. I am someone who is open to new experiences. 10. I am someone who is open-minded and curious about different cultures.

1. I am someone who finds books dull.

2. I am someone who has difficulty interpreting and applying abstract concepts.

3. I am someone who has little curiosity about the nature of things.

4. I am someone who has little interest in art and aesthetics.

5. I am someone who thinks philosophy is a useless waste of time.

6. I am someone who has little intellectual curiosity.

7. I am someone who tends to reject ideas that are contradictory to my own opinions. 
Iteration 3

(Conscientiousness, regular-keyed)

Iteration 4

(Conscientiousness, reverse-keyed)

Iteration 5

(Extraversion, regular-keyed)

Iteration 6

(Extraversion, reverse-keyed)

Iteration 7

(Agreeableness, regular-keyed)
1. I am someone who has a strong work ethic and can be counted on to get things done.

2. I am someone who is self-disciplined, works hard.

3. I am someone who gets the job done, even when it is a bit complicated.

4. I am someone who looks after details, is diligent.

5. I am someone who is organized, keeps things tidy.

6. I am someone who is meticulous and precise.

1. I am someone who is usually inattentive.

2. I am someone who is disorganized and prone to losing track of things.

3. I am someone who is often late.

4. I am someone who tends to get distracted.

5. I am someone who can be unreliable.

6. I am someone who is prone to being messy.

1. I am someone who is excitable.

2. I am someone who likes to talk.

3. I am someone who likes to interact with people.

4. I am someone who makes friends easily.

5. I am someone who is very energetic.

6. I am someone who has an outgoing personality.

7. I am someone who loves to be social.

8. I am someone who has lots of friends.

9. I am someone who likes to entertain.

1. I am someone who prefers to be just with the people who are close to me.

2. I am someone who avoids social gatherings.

3. I am someone who is awkward at social situations.

4. I am someone who enjoys solitude.

5. I am someone who would prefer to let others handle the situation.

6. I am someone who is not very talkative.

7. I am someone who is often anxious about having conversations with strangers.

8. I am someone who feels uncomfortable in social situations.

1. I am someone who is easy to get along with.

2. I am someone who is patient and kind.

3. I am someone who is kind and compassionate towards others.

4. I am someone who values friendship and holds it dear.

5. I am someone who does not judge others.

6. I am someone who helps others.

7. I am someone who is tolerant and understanding.

8. I am someone who sees the good in everyone.

9. I am someone who gives the benefit of the doubt.

10. I am someone who is sensitive to others needs.

11. I am someone who is amiable, tender and kind to others. 
Iteration 8

(Agreeableness, reverse-keyed)

Iteration 9

(Neuroticism, regular-keyed)

Iteration 10

(Neuroticism, reverse-keyed)
12. I am someone who is trusting.

13. I am someone who cares a lot about the wellbeing of others.

1. I am someone who makes others feel inferior.

2. I am someone who gets angry easily.

3. I am someone who can be impolite and disrespectful.

4. I am someone who is easily annoyed.

5. I am someone who spends a lot of time and energy arguing.

6. I am someone who is slow to apologize.

7. I am someone who doesn't often trust the opinions of others.

8. I am someone who can be difficult to be around.

9. I am someone who holds grudges.

10. I am someone who can be distant and mean.

11. I am someone who is slow to forgive others.

12. I am someone who is quick to condemn others.

13. I am someone who is quick to criticize others.

1. I am someone who seems to keep things bottled up.

2. I am someone who often feels anxious.

3. I am someone who can be moody.

4. I am someone who feels stressed out.

5. I am someone who is easily irritable.

6. I am someone who is easily triggered.

7. I am someone who can be insecure.

8. I am someone who is volatile.

9. I am someone who has trouble coping with stress.

10. I am someone who cannot relax.

1. I am someone who can handle uncertainty.

2. I am someone who is able to cope with anything.

3. I am someone who is able to work through challenges successfully.

4. I am someone who is not easily bothered or upset.

5. I am someone who is calm and collected under stressful situations.

6. I am someone who has a relaxed outlook on life.

7. I am someone who feels at ease and composed.

8. I am someone who does not panic easily.

9. I am someone who can accept and overcome challenges.

10. I am someone who keeps a positive attitude.

Note. Italicised items were selected by three of the four members of the expert committee. All other items reached unanimous consensus. Bold items were selected for inclusion in the final N-BFI-20 (STEP 3). 
In STEP 3, we recruited a gender-balanced sample of 512 participants via Prolific Academic (https://www.prolific.co/), an established crowd-working platform which provides competitive data quality (Palan \& Schitter, 2018; Peer et al., 2017). In order to take part in the study, participants needed to be at least 18 years of age, and had to reside in Canada at the time of participating in the research. After excluding 11 individuals who a) participated in the study multiple times, b) indicated being below 18 years old or above 120 years old, c) did not give consent to participate, or d) did not complete the full study, our final sample consisted of 501 participants $\left(\operatorname{age}_{M}=31.18\right.$, age $_{S D}=10.90 ; 47.7 \%$ female, $48.7 \%$ male, $2.2 \%$ non-binary, $1.0 \%$ other, $0.4 \%$ prefer not to say). The data collection was carried out in November 2021. Survey completion took approximately 10 minutes and participants received a compensation of $£ 1.50$ (i.e., approximately US\$1.81, CA\$2.33), in keeping with Prolific's ethical payment principles and current minimum wage standards in the Canadian province of British Columbia.

As part of the study, all participants completed all items from the N-BFI pool formed in STEP 2 (see Table 3), using a Likert-scale ranging from 1 ("Disagree strongly") to 7 (“Agree strongly”). In addition, all participants self-reported their age, gender, and income. To extract the final N-BFI-20 from the larger set of candidate items, we adopted a multicriteria approach based on exploratory factor analyses (EFAs) and item response theory (IRT).

In a first preparatory step, applying the Kaiser-Meyer-Olkin (KMO) Measure of Sampling Adequacy and Bartlett's Test of Sphericity suggested that our data were very wellsuited for factor analysis (Bartlett's $\chi^{2}=27876.69, d f=4186, p<.001 ; \mathrm{KMO}=.925$; Carpenter, 2018; Tabachnick \& Fidell, 2007). Next, we ran exploratory factor analyses with a pre-specified five factor solution, in keeping with our top-down item development process and the theoretical rationale underlying the Big Five taxonomy. Following previous research 
and established psychometrics guidelines, upon inspecting the resulting factor matrices, we first removed all items that showed primary factor loadings weaker than .40 (Clark \& Watson, 2019; Ford et al., 1986; Hair et al., 2010; Rosellini \& Brown, 2021), and/or crossloadings above .30 (Boateng et al., 2018; Costello \& Osborne, 2005). In a first iteration this process erased 22 items from the item pool. We then repeated the procedure until no items remained that fit these exclusion criteria (iteration 2: five items; iteration 3: two items; iteration 4: zero items). Second, we excluded all items with communalities smaller than .30 (Carpenter, 2018; Fabrigar et al., 1999; Worthington \& Whittaker, 2006), removing another 6 items. ${ }^{6}$ After these generic EFA-based steps, we examined each sub-scale (i.e., Openness, Conscientiousness, Extraversion, Agreeableness, Neuroticism) separately and implemented the remaining exclusion criteria within the context of individual subscales. That is, we fitted multidimensional 2-parameter IRT models for each subscale. We then first conducted differential item functioning (DIF) analyses (Holland \& Wainer, 1993; Nguyen et al., 2014; Reise et al., 1993) to examine whether items yielded the same measurement properties across different groups (Flake et al., 2017; Millsap, 2011). Specifically, we investigated whether items functioned differently across gender- (men vs women) as well as age-groups (high vs low, based on a median split within the overall sample). At this stage, nine items showed evidence of differential item functioning and were hence removed (Openness: four items; Conscientiousness: zero items; Extraversion: three items; Agreeableness: two items; Neuroticism: zero items). As a last step we conducted item information function (IIF) analyses, as well as test information function (TIF) analyses to guide final item selections. Therein, our goal was to select a set of four overall items per dimension (each two regularkeyed and two reverse-keyed) which would jointly cover a broad range of item difficulty

\footnotetext{
${ }^{6} \mathrm{We}$ had pre-registered the more conservative criterion to remove items with communalities lower than .40 . However, as this resulted in the removal of all reverse-keyed items for Agreeableness, we relaxed this criterion and removed items with communalities lower than .30 instead. See also the next paragraph for more details about the challenges related to reverse-keyed items.
} 
levels and in turn allow to discriminate between individuals across the whole respective trait spectrum (Anastasi \& Urbina, 1997; Bock et al., 1988; Clifton, 2020).

Carrying out these steps enabled us to create key-balanced four-item sub-scales for four of the five Big Five traits. However, employing our stringent criteria meant that no reverse-keyed Openness item would be eligible for inclusion. Importantly, this is not a new phenomenon and others have encountered the same challenge before us. Indeed, Big Five Openness scales have a history of being heavily skewed in favour of regular-, rather than reverse-keyed items (Danner et al., 2015; John \& Srivastava, 1999), with some scales even failing to encompass any reverse-keyed items (Soto \& John, 2009). Leading scholars in the field have attributed this to the fact that in natural language the vast majority of adjectives describing personal openness refer to high, rather than low levels of openness (Hofstee et al., 1992; John, 1989, 1990; Soto \& John, 2017). More generally, there is a broad agreement in the field that reverse-keyed items are more likely to fare poorly within the set of traditional selection criteria — and hence be excluded from scales — than their regular-keyed counterparts (Clifton, 2020; DeVellis, 2003). However, retaining reverse-keyed items is of critical importance to ensure that scales can distinguish between meaningful personality information and response biases reflecting indiscriminate acquiescence (yea-saying; Clifton, 2020; Cronbach, 1946; Jackson \& Messick, 1958; Paulhus, 1991; Rammstedt \& Danner, 2017; Soto \& John, 2017). Moreover, reverse-keyed items allow wider domain coverage (Clark \& Watson, 1995; Clifton, 2020). Against this backdrop, we decided to selectively relax our exclusion criteria by allowing items with factor loadings $<.20$ for Openness in order to retain two reverse-keyed Openness items for the sake of achieving within- and between-sub-scale content balance on the N-BFI-20. The final 20 items that comprise the N-BFI-20 (four per personality dimension) are highlighted in bold in Table 3. 
For full details on each analytical step and complete results, we refer readers to an annotated R Markdown file which we provide on our Open Science Framework project page (https://osf.io/fhpv6/?view only=c7d15f8becaf4ee3b8b7e59aba4de325; blinded for review).

\section{STEP 4: Confirmatory Study I-Validation of the Neural-BFI-20}

In STEP 4, we recruited a sample of college students from the University of British Columbia, a large public university in Western Canada. An a priori power analysis conducted in G*Power 3.1 (Faul et al., 2007, 2009) indicated that we would need to recruit 447 participants in order to reach a statistical power of 0.80 to detect correlations of $r=0.10$ (Funder \& Ozer, 2019; Gignac \& Szodorai, 2016). We thus conservatively aimed to recruit at least 500 participants and ultimately recruited 914, of which 773 were included in our analyses after applying the following exclusion criteria: a) reported being less than 18 or more than 120 years old, b) did not give consent to participate, c) did not complete the full study, or d) completed the study multiple times. Of these, 557 (72.1\%) identified as female, $201(26.0 \%)$ identified as male, $11(1.4 \%)$ identified as non-binary, $1(0.1 \%)$ as other, and 3 $(0.4 \%)$ preferred not to disclose their gender — which is broadly representative of the undergraduate student population at the Department of Psychology at the University of British Columbia. As participants were undergraduate students, the sample was young $\left(M d n_{\text {age }}=19.00, M_{\text {age }}=20.09, S D_{\text {age }}=2.43\right)$.

The data collection was carried out in late November and early December 2021. Survey completion took approximately 25 minutes and participants received course credit for their participation. As part of the survey study, all participants completed the newlydeveloped N-BFI-20, along with the original BFI-2 (Soto \& John, 2017). In addition, the survey assessed sociodemographic information (i.e., age, gender) as well as the following psychological constructs that had been used in previous Big Five scale validations (Donnellan et al., 2006; Gosling et al., 2003) and which were used in the current study to 
create a nomological network and establish construct validity: social dominance orientation (using the $\mathrm{SDO}_{7}$ scale; Ho et al., 2015; Pratto et al., 1994), personal music preferences (using the short test of music preferences (STOMP); Rentfrow \& Gosling, 2003), and self-esteem (using the single-item self-esteem measure; Robins et al., 2001).

In a first step, we assessed basic psychometric properties. That is, we calculated McDonald's $\omega$ (Dunn et al., 2014; McDonald, 1999; Revelle \& Condon, 2019) to gauge reliability and probed structural validity by conducting confirmatory factor analyses on both the N-BFI-20 and the BFI-2. The $\omega$ values are slightly higher for the 60 -item BFI-2 $\left(\omega_{\mathrm{o}}=0.87, \omega_{\mathrm{c}}=0.83\right.$, $\left.\omega_{\mathrm{e}}=0.86, \omega_{\mathrm{a}}=0.82, \omega_{\mathrm{n}}=0.88\right)$ than for the 20-item N-BFI-20 $\left(\omega_{\mathrm{o}}=0.63, \omega_{\mathrm{c}}=0.71, \omega_{\mathrm{e}}=\right.$ $\left.0.81, \omega_{\mathrm{a}}=0.64, \omega_{\mathrm{n}}=0.74\right)$-which is typical for parsimonious scales that emphasise construct breadth and seek to avoid redundancy (Clifton, 2020; Gosling et al., 2003; Rammstedt \& John, 2007) — but both show acceptable reliability or better for each of the personality traits. When evaluating fit against conventional guidelines (RMSEA/SRMR $<.10$ $=$ acceptable $;<.06=$ excellent $;$ CFI $/$ TLI $>.90=$ acceptable $;>.95=$ excellent Clark $\&$ Watson, 2019; Finch \& West, 1997; Hu \& Bentler, 1999; Pituch \& Stevens, 2015; Schumacker et al., 2015), for the N-BFI-20, the model indicated acceptable fit for the RMSEA (.090, 90\% CI [.084, .095]) and SRMR (0.092) criteria and insufficient fit for the CFI (.77) and TLI (.73) criteria. We also note that the $\chi^{2}$ goodness of fit test reached significance, thus suggesting lack of fit $\left(\chi^{2}=958.39, p<.001\right)$.

Similarly, for the BFI-2, acceptable fit was achieved for the RMSEA $(.074,90 \%$ CI $[.073, .076])$ criterion, whereas insufficient fit was found for the SRMR (0.100), CFI (.63) and TLI (.61). As was the case for the N-BFI-20, the $\chi^{2}$ goodness of fit for the BFI-2 was significant, indicating lack of fit $\left(\chi^{2}=7441.61, p<.001\right)$. While puzzling at first glance, the comparatively weak support for the structural validity of both the N-BFI-20 and the BFI-2 based on CFAs is actually very well aligned with the broader scientific literature. In fact- 
across research teams and Big Five instruments - failure to support Big Five measures that have well-defined EFA structures through CFA metrics has been so commonplace that a recent review did not find a single CFA conducted at the item level that offered acceptable support for the a-priori Big Five factor structure (Marsh et al., 2010). Accordingly, for several decades, personality psychologists across the field have questioned the appropriateness of CFAs to evaluate the factorial structure of personality constructs in general and the Big Five in particular and argued — and showed — that the typical independent clusters models (ICM) used in CFA studies are indeed overly restrictive (Borkenau \& Ostendrof, 1990; Church \& Burke, 1994; Hopwood \& Donnellan, 2010; McCrae et al., 1996; Parker et al., 1993; Vassend \& Skrondal, 1997). Building on this, Marsh et al. (2010, 2013) have used empirical Big Five-based demonstrations to advocate for the use of exploratory structural equation modelling approaches (ESEM; Asparouhov \& Muthén, 2009; Marsh et al., 2009, 2014), which combine the most desirable aspects of EFAs and CFAs by offering the many methodological advances afforded by CFAs without the overly restrictive assumptions of traditional ICM-CFA approaches. Since then, ESEM has largely replaced traditional CFAs in the assessment of the structural validity of Big Five measures (e.g., Chiorri et al., 2016; Lang et al., 2011; Rammstedt et al., 2020, 2021).

Against this backdrop, we deemed it appropriate to deviate from our pre-registration and additionally run ESEM analyses to scrutinise the structural validity of both, the N-BFI-20 and the BFI-2. ESEM analyses yielded good—and for some metrics even excellent—-fit properties for the N-BFI $20($ RMSEA $=.058,90 \%$ CI $[.051, .065], \mathrm{SRMR}=.030, \mathrm{CFI}=.94$, $\mathrm{TLI}=.89)^{7}$

\footnotetext{
${ }^{7}$ While markedly improved on the RMSEA $(.061,90 \%$ CI $[.060, .063])$ and SRMR $(.047)$ metrics, initially the BFI-2 (Soto \& John, 2017) continued to yield sub-optimal fit metrics on the CFI (.78), TLI (.73) metrics. However, upon accounting for the complex facet-level structure of the BFI-2 and explicitly modelling it, the fit indices improved substantially and consistently indicated good to excellent fit (RMSEA = .037, SRMR $=.016$, $\mathrm{CFI}=.96, \mathrm{TLI}=.91)$.
} 
Having thus established the structural validity of the N-BFI-20, in a second step, we set out to examine the nomological network of the N-BFI-20 and its relationship with the BFI-2, to further gauge construct validity. In keeping with previous research, we expectedand pre-registered - the following relationship patterns:

H1: Each Big Five dimension of the N-BFI-20 will be most strongly positively correlated with the respective identical dimension in the BFI-2.

H2: Social dominance orientation will be negatively correlated with Agreeableness and Openness in both the Neural-BFI-20 and the BFI-2 (Gosling et al., 2003).

H3: The Big Five will be correlated with music preferences in both the Neural-BFI-20 and the BFI-2. Specifically:

H3a: A preference for reflective and complex music will be positively correlated with Openness (Gosling et al., 2003).

H3b: A preference for intense and rebellious music will be positively correlated with Openness (Gosling et al., 2003).

H3c: A preference for upbeat and conventional music will be positively correlated with Conscientiousness, Extraversion, and Agreeableness (Gosling et al., 2003).

H3d: A preference for energetic and rhythmic music will be positively correlated with Extraversion (Gosling et al., 2003).

H4: Self-esteem will be positively correlated with Openness, Conscientiousness, Extraversion, and Agreeableness, as well as negatively correlated with Neuroticism in both the Neural-BFI-20 and the BFI-2 (Donnellan et al., 2006; Gosling et al., 2003).

As predicted in H1, across the two Big Five measures (i.e., N-BFI-20, BFI-2), each trait was most strongly positively associated with the same trait in the respective other measure (Openness: $r=.731, p<.001$; Conscientiousness: $r=.821, p<.001$; Extraversion: $r$ $=.795, p<.001$; Agreeableness: $r=.767, p<.001$; Neuroticism: $r=.786, p<.001$; see 
Table 4). In addition, the N-BFI-20 largely reproduced inter-trait correlation patterns observed in the BFI-2. To illustrate, across both measures, Openness showed small-tomedium-sized positive correlations with Conscientiousness (N-BFI-20: $r=.091, p=.011$; BFI-2: $r=.130, p<.001)$, Extraversion (N-BFI-20: $r=.267, p<.001 ;$ BFI-2: $r=.291, p$ $<.001$ ), and Agreeableness (N-BFI-20: $r=.397, p<.001 ; \mathrm{BFI}-2: r=.356, p<.001)$;

Conscientiousness showed small-to-medium-sized positive correlations with Extraversion $(\mathrm{N}-\mathrm{BFI}-20: r=.123, p<.001 ; \mathrm{BFI}-2: r=.211, p<.001)$ and Agreeableness (N-BFI-20: $r$ $=.190, p<.001 ;$ BFI-2: $r=.364, p<.001) ;$ Extraversion showed small positive correlations with Agreeableness (N-BFI-20: $r=.103, p=.004$; BFI-2: $r=.124, p<.001)$ and mediumsized negative correlations with Neuroticism (N-BFI-20: $r=-.236, p<.001$; BFI-2: $r=-.328$, $p<.001)$.

Table 4

Correlations Between the N-BFI-20 and the BFI-2

\begin{tabular}{|c|c|c|c|c|c|c|}
\hline & & \multicolumn{2}{|c|}{ Pearson's $r$} & \multirow[t]{2}{*}{$p$} & \multicolumn{2}{|c|}{$95 \% \mathrm{CI}$} \\
\hline & & & & & $L L$ & $U L$ \\
\hline N-BFI-20 O & - $\quad$ BFI-2 O & 0.731 & $* * *$ & $<.001$ & 0.696 & 0.762 \\
\hline N-BFI-20 C & - $\quad$ BFI-2 C & 0.821 & $* * *$ & $<.001$ & 0.796 & 0.843 \\
\hline N-BFI-20 E & - BFI-2 E & 0.795 & $* * *$ & $<.001$ & 0.768 & 0.820 \\
\hline N-BFI-20 A & - BFI-2 A & 0.767 & $* * *$ & $<.001$ & 0.736 & 0.794 \\
\hline N-BFI-20 N & - $\quad$ BFI-2 N & 0.786 & $* * *$ & $<.001$ & 0.758 & 0.812 \\
\hline
\end{tabular}

Note. Bold cells represent a significant correlation above $r=0.700 . N=773 . \mathrm{O}=$ Openness. $\mathrm{C}=$

Conscientiousness. $\mathrm{E}=$ Extraversion $. \mathrm{A}=$ Agreeableness. $\mathrm{N}=$ Neuroticism $. \mathrm{CI}=$ confidence interval. $L L=$ lower limit. $U L=$ upper limit. $* * * p<.001, * * p<.01, * p<.05$

Contrary to H2, in both the N-BFI-20 and the BFI-2 neither Openness (N-BFI-20: $r=$ $-.036, p=.730 ;$ BFI-2: $r=-.139, p=.178)$, nor Agreeableness $(\mathrm{N}-\mathrm{BFI}-20: r=-.089, p$ $=.389 ; \mathrm{BFI}-2: r=-.166, p=.105)$ were significantly negatively related to social dominance orientation. Instead, in both the N-BFI-20 and the BFI-2, Extraversion was positively correlated with social dominance orientation (N-BFI-20: $r=.339, p<.001$; BFI-2: $r=.346, p$ $<.001)$. 
In terms of music preferences, in line with $\mathrm{H} 3 \mathrm{a}$, Openness was positively correlated with a preference for reflective and complex music (N-BFI-20: $r=.156, p<.001$; BFI-2: $r$ $=.249, p<.001)$ in both the N-BFI-20 and the BFI-2. In line with H3b, Openness was further positively correlated with a preference for intense and rebellious music (N-BFI-20: $r=.179$, $p<.001 ; \mathrm{BFI}-2: r=.195, p<.001)$ in both the N-BFI-20 and the BFI-2. In line with H3c, Extraversion was positively correlated with a preference for upbeat and conventional music $(\mathrm{N}-\mathrm{BFI}-20: r=.102, p=.005 ; \mathrm{BFI}-2: r=.095, p=.008)$ in both the N-BFI-20 and the BFI-2. However, contrary to $\mathrm{H} 3 \mathrm{c}$, in both the N-BFI-20 and the BFI-2 neither Conscientiousness (NBFI-20: $r=.058, p=.110 ; \mathrm{BFI}-2: r=.070, p=.052)$, nor Agreeableness (N-BFI-20: $r$ $=.039, p=.279 ; \mathrm{BFI}-2: r=.055, p=.130$ ) were significantly positively associated with a preference for upbeat and conventional music.

Lastly, in keeping with H3d, Extraversion was positively correlated with a preference for energetic and rhythmic music (N-BFI-20: $r=.142, p<.001$; BFI-2: $r=.164, p<.001)$ in both the N-BFI-20 and the BFI-2.

Supporting H4, in both the N-BFI-20 and the BFI-2, Conscientiousness (N-BFI-20: $r$ $=.105, p=.004 ; \mathrm{BFI}-2: r=.119, p<.001)$, and Extraversion $(\mathrm{N}-\mathrm{BFI}-20: r=.335, p<.001$; BFI-2: $r=.405, p<.001$ ) were positively correlated with self-esteem, whereas Neuroticism was negatively correlated with self-esteem (N-BFI-20: $r=-.467, p<.001 ; \mathrm{BFI}-2: r=-.520, p$ $<.001)$. In addition, not supporting H4, Openness was significantly positively correlated with self-esteem in neither the N-BFI-20 $(r=.022, p=.544)$ nor the BFI-2 $(r=.022, p=.544)$. Also contrary to $\mathrm{H} 4$, Agreeableness was not positively associated with self-esteem in either of the two Big Five measures (N-BFI-20: $r=-.084, p=.019$; BFI-2: $r=-.042, p=.244$ ).

Table 5 shows a correlation table exhibiting the associations between the Big Five traits across the N-BFI-20 and the BFI-20, as well as how the Big Five traits in both measures relate to the other variables in the nomological net, highlighting their similarities and 
differences, with $95 \%$ confidence intervals. Figure 1 displays the full correlation matrix, detailing bivariate relationships for all variables in the dataset. 
Table 5

Internal and External Correlation Patterns of the N-BFI-2 and the BFI-2

\begin{tabular}{|c|c|c|c|c|c|c|c|c|c|c|c|}
\hline & & \multicolumn{5}{|c|}{ N-BFI-20 } & \multicolumn{5}{|c|}{ BFI-2 } \\
\hline & & \multirow{2}{*}{\multicolumn{2}{|c|}{ Pearson's $r$}} & \multirow[t]{2}{*}{$p$} & \multicolumn{2}{|c|}{$95 \% C I$} & \multicolumn{2}{|c|}{ Pearson's $r$} & \multirow[t]{2}{*}{$p$} & \multicolumn{2}{|c|}{$95 \% C I$} \\
\hline & & & & & $L L$ & $U L$ & & & & $L L$ & $U L$ \\
\hline \multicolumn{12}{|c|}{ INTERNAL CORRELATIONS } \\
\hline Openness & - Conscientiousness & 0.091 & $*$ & 0.011 & 0.021 & 0.161 & 0.130 & $* * *$ & $<.001$ & 0.060 & 0.199 \\
\hline Openness & - Extraversion & 0.267 & $* * *$ & $<.001$ & 0.201 & 0.332 & 0.291 & $* * *$ & $<.001$ & 0.225 & 0.354 \\
\hline Openness & - Agreeableness & 0.397 & $* * *$ & $<.001$ & 0.335 & 0.454 & 0.356 & $* * *$ & $<.001$ & 0.293 & 0.417 \\
\hline Openness & - Neuroticism & -0.068 & & 0.060 & -0.138 & 0.003 & 0.048 & & 0.183 & -0.023 & 0.118 \\
\hline Conscientiousness & - Extraversion & 0.123 & $* * *$ & $<.001$ & 0.053 & 0.192 & 0.211 & $* * *$ & $<.001$ & 0.142 & 0.277 \\
\hline Conscientiousness & - Agreeableness & 0.190 & $* * *$ & $<.001$ & 0.121 & 0.257 & 0.364 & $* * *$ & $<.001$ & 0.302 & 0.424 \\
\hline Conscientiousness & - Neuroticism & -0.049 & & 0.169 & -0.120 & 0.021 & -0.209 & $* * *$ & $<.001$ & -0.275 & -0.140 \\
\hline Extraversion & - Agreeableness & 0.103 & $* *$ & 0.004 & 0.033 & 0.173 & 0.124 & $* * *$ & $<.001$ & 0.054 & 0.193 \\
\hline Extraversion & - Neuroticism & -0.236 & $* * *$ & $<.001$ & -0.301 & -0.168 & -0.328 & $* * *$ & $<.001$ & -0.389 & -0.263 \\
\hline Agreeableness & - Neuroticism & -0.007 & & 0.853 & -0.077 & 0.064 & -0.064 & & 0.073 & -0.134 & 0.006 \\
\hline \multicolumn{12}{|c|}{ EXTERNAL CORRELATIONS } \\
\hline Openness & - Age & -0.020 & & 0.579 & -0.090 & 0.051 & 0.000 & & 0.995 & -0.070 & 0.071 \\
\hline Openness & - Gender: Male & -0.153 & $* * *$ & $<.001$ & -0.222 & -0.083 & -0.143 & $* * *$ & $<.001$ & -0.212 & -0.072 \\
\hline Openness & - Self-Esteem & 0.058 & & 0.106 & -0.012 & 0.128 & 0.022 & & 0.544 & -0.049 & 0.092 \\
\hline Openness & - SDO & -0.036 & & 0.730 & -0.235 & 0.166 & -0.139 & & 0.178 & -0.330 & 0.064 \\
\hline Openness & - STOMP Reflective & 0.156 & $* * *$ & $<.001$ & 0.086 & 0.224 & 0.249 & $* * *$ & $<.001$ & 0.181 & 0.314 \\
\hline Openness & - STOMP Intense & 0.179 & $* * *$ & $<.001$ & 0.110 & 0.247 & 0.195 & $* * *$ & $<.001$ & 0.126 & 0.262 \\
\hline Openness & - STOMP Upbeat & -0.002 & & 0.950 & -0.073 & 0.068 & 0.052 & & 0.153 & -0.019 & 0.122 \\
\hline Openness & - STOMP Energetic & 0.142 & $* * *$ & $<.001$ & 0.072 & 0.211 & 0.137 & $* * *$ & $<.001$ & 0.067 & 0.205 \\
\hline Conscientiousness & - Age & 0.006 & & 0.864 & -0.064 & 0.077 & -0.002 & & 0.966 & -0.072 & 0.069 \\
\hline Conscientiousness & - Gender: Male & -0.066 & & 0.068 & -0.137 & 0.005 & -0.089 & $*$ & 0.014 & -0.159 & -0.018 \\
\hline Conscientiousness & - Self-Esteem & 0.105 & $* *$ & 0.004 & 0.035 & 0.174 & 0.119 & $* * *$ & $<.001$ & 0.048 & 0.188 \\
\hline Conscientiousness & - SDO & 0.166 & & 0.106 & -0.036 & 0.355 & 0.165 & & 0.109 & -0.037 & 0.354 \\
\hline Conscientiousness & - STOMP Reflective & -0.088 & $*$ & 0.015 & -0.158 & -0.017 & -0.041 & & 0.255 & -0.112 & 0.030 \\
\hline
\end{tabular}




\begin{tabular}{|c|c|c|c|c|c|c|c|c|c|c|c|}
\hline Conscientiousness & - STOMP Intense & -0.154 & $* * *$ & $<.001$ & -0.222 & -0.085 & -0.115 & $* *$ & 0.001 & -0.184 & -0.045 \\
\hline Conscientiousness & - STOMP Upbeat & 0.058 & & 0.110 & -0.013 & 0.128 & 0.070 & & 0.052 & -0.001 & 0.140 \\
\hline Conscientiousness & - $\quad$ STOMP Energetic & -0.024 & & 0.511 & -0.094 & 0.047 & -0.029 & & 0.428 & -0.099 & 0.042 \\
\hline Extraversion & - Age & 0.029 & & 0.417 & -0.041 & 0.100 & 0.022 & & 0.546 & -0.049 & 0.092 \\
\hline Extraversion & - Gender: Male & 0.025 & & 0.488 & -0.046 & 0.096 & 0.056 & & 0.125 & -0.015 & 0.127 \\
\hline Extraversion & - Self-Esteem & 0.335 & $* * *$ & $<.001$ & 0.271 & 0.396 & 0.405 & $* * *$ & $<.001$ & 0.344 & 0.462 \\
\hline Extraversion & - SDO & 0.339 & $* * *$ & $<.001$ & 0.148 & 0.505 & 0.346 & $* * *$ & $<.001$ & 0.156 & 0.511 \\
\hline Extraversion & - $\quad$ STOMP Reflective & 0.079 & $*$ & 0.028 & 0.009 & 0.149 & 0.087 & $*$ & 0.015 & 0.017 & 0.157 \\
\hline Extraversion & - $\quad$ STOMP Intense & 0.059 & & 0.100 & -0.011 & 0.129 & 0.049 & & 0.174 & -0.022 & 0.119 \\
\hline Extraversion & - STOMP Upbeat & 0.102 & $* *$ & 0.005 & 0.031 & 0.171 & 0.095 & $* *$ & 0.008 & 0.025 & 0.165 \\
\hline Extraversion & - $\quad$ STOMP Energetic & 0.142 & $* * *$ & $<.001$ & 0.072 & 0.211 & 0.164 & $* * *$ & $<.001$ & 0.095 & 0.232 \\
\hline Agreeableness & - Age & -0.015 & & 0.679 & -0.085 & 0.056 & -0.005 & & 0.888 & -0.076 & 0.065 \\
\hline Agreeableness & - Gender: Male & -0.187 & $* * *$ & $<.001$ & -0.254 & -0.117 & -0.220 & $* * *$ & $<.001$ & -0.287 & -0.151 \\
\hline Agreeableness & - Self-Esteem & -0.084 & $*$ & 0.019 & -0.154 & -0.014 & -0.042 & & 0.243 & -0.112 & 0.029 \\
\hline Agreeableness & - SDO & -0.089 & & 0.389 & -0.284 & 0.114 & -0.166 & & 0.105 & -0.355 & 0.035 \\
\hline Agreeableness & - STOMP Reflective & 0.004 & & 0.912 & -0.067 & 0.075 & 0.004 & & 0.919 & -0.067 & 0.074 \\
\hline Agreeableness & - STOMP Intense & 0.026 & & 0.469 & -0.045 & 0.097 & 0.018 & & 0.625 & -0.053 & 0.088 \\
\hline Agreeableness & - STOMP Upbeat & 0.039 & & 0.279 & -0.032 & 0.109 & 0.055 & & 0.130 & -0.016 & 0.125 \\
\hline Agreeableness & - $\quad$ STOMP Energetic & 0.008 & & 0.814 & -0.062 & 0.079 & 0.000 & & 0.994 & -0.071 & 0.070 \\
\hline Neuroticism & - Age & -0.078 & $*$ & 0.030 & -0.148 & -0.008 & -0.057 & & 0.114 & -0.127 & 0.014 \\
\hline Neuroticism & - Gender: Male & -0.273 & $* * *$ & $<.001$ & -0.338 & -0.206 & -0.261 & $* * *$ & $<.001$ & -0.326 & -0.193 \\
\hline Neuroticism & - Self-Esteem & -0.467 & $* * *$ & $<.001$ & -0.520 & -0.410 & -0.520 & $* * *$ & $<.001$ & -0.570 & -0.467 \\
\hline Neuroticism & - SDO & -0.014 & & 0.896 & -0.213 & 0.187 & -0.132 & & 0.199 & -0.324 & 0.070 \\
\hline Neuroticism & - STOMP Reflective & -0.028 & & 0.437 & -0.099 & 0.043 & 0.013 & & 0.720 & -0.058 & 0.084 \\
\hline Neuroticism & - $\quad$ STOMP Intense & -0.002 & & 0.961 & -0.072 & 0.069 & 0.062 & & 0.084 & -0.008 & 0.132 \\
\hline Neuroticism & - STOMP Upbeat & -0.070 & & 0.052 & -0.140 & 0.001 & -0.053 & & 0.142 & -0.123 & 0.018 \\
\hline Neuroticism & - $\quad$ STOMP Energetic & -0.059 & & 0.104 & -0.129 & 0.012 & -0.008 & & 0.825 & -0.078 & 0.063 \\
\hline
\end{tabular}

Note. Green cells represent agreement between the N-BFI-20 and the BFI-2, orange cells represent disagreement. $N=773$. CI $=$ confidence interval. $L L=$ lower limit. $U L=$ upper limit. $* * * p<.001, * * p<.01, * p<.05$ 


\section{Figure 1}

Bivariate Correlation Matrix - University of British Columbia Sample

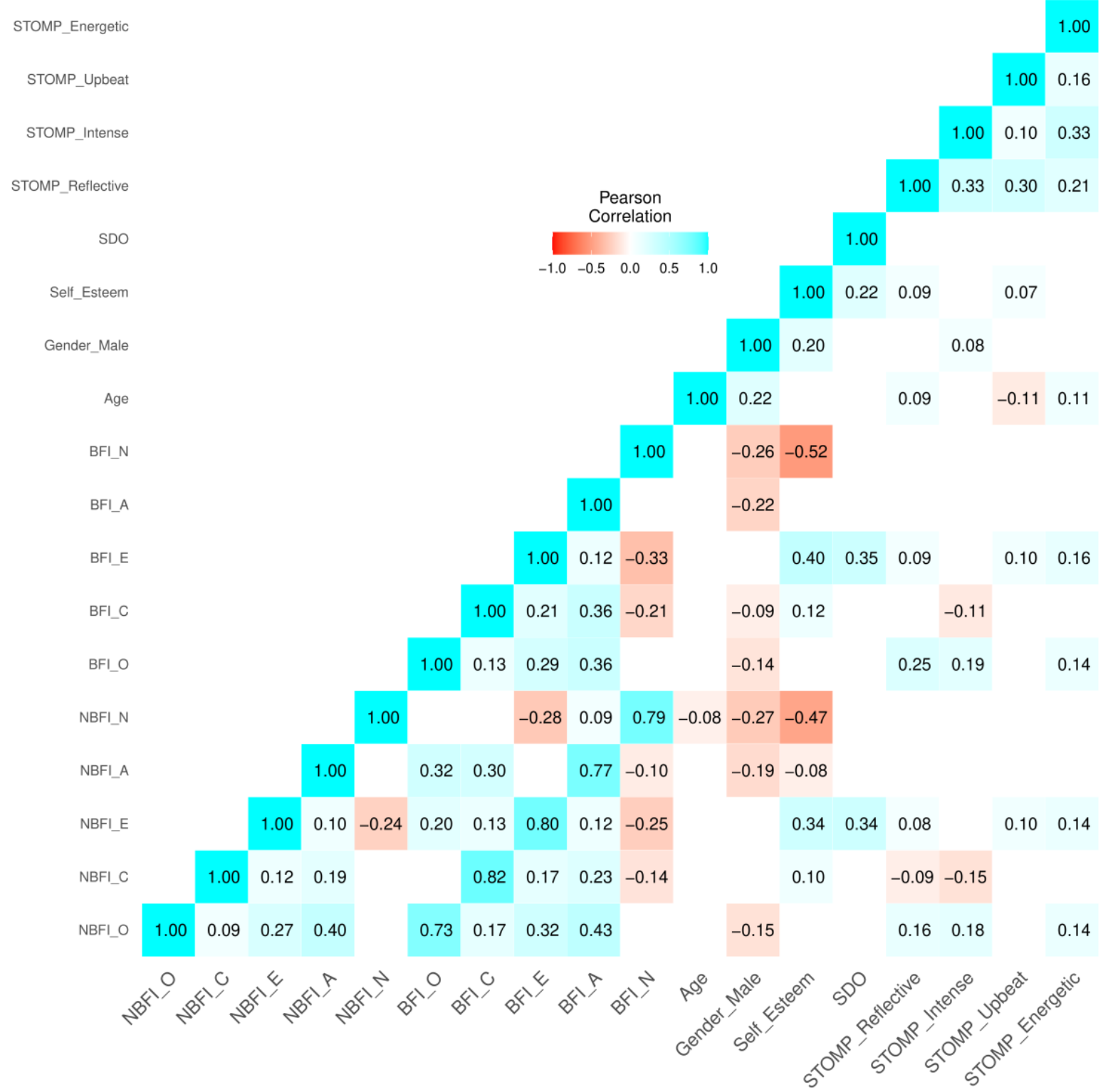

\section{STEP 5: Confirmatory Study II-Validation of the Neural-BFI-20}

In the fifth and last step, we revisited the Prolific sample recruited in STEP 3 to readminister the N-BFI-20, assess its test-retest reliability and probe its predictive validity (Clifton, 2020). In keeping with previous research (Gosling et al., 2003; Donnellan et al., 2006) and recommended practices (Watson, 2004) we made sure that for all participants a minimum interval of three weeks lay between their initial completion of the N-BFI and the 
re-administration $\left(Q_{1}=24\right.$ days, $Q_{3}=24$ days). Of the original 501 participants (see STEP 3), 449 completed the follow-up survey $\left(\operatorname{age}_{M}=31.46\right.$, age $S D=11.10 ; 47.8 \%$ female, $49.1 \%$ male, $1.6 \%$ non-binary, $1.1 \%$ other, $0.4 \%$ preferred not to disclose their gender; income $M d n=$ $37,957 \mathrm{CA} \$, 67.3 \%$ with postsecondary degree), corresponding to an overall retention rate of $89.4 \%$, which is consistent with — and if anything slightly higher than — retention rates in previous research (Palan \& Schitter, 2018; Maertens et al., 2021; Zielinski \& Veilleux, 2018). Survey completion took approximately 5 minutes and participants received a compensation of $£ 0.75$ (i.e., approximately US\$ 0.91, CA\$ 1.16), in keeping with Prolific's ethical payment principles and current minimum wage standards in the Canadian province of British Columbia. In addition to having participants retake the N-BFI-20 - paralleling previous Big Five scale validation research (Rammstedt et al., 2020)—we also assessed personal health status (five-point Likert-scale anchored at $1=$ poor, 5 =excellent; Rammstedt et al., 2020), life satisfaction (12-point Likert scale anchored at $0=$ not satisfied at all; $11=$ completely satisfied; single-item life satisfaction scale, Beierlein et al., 2014), educational attainment (highest completed level of education; anchored at no formal education, doctoral degree), annual income in CA $\$$ and political orientation (seven-point scale; anchored at $1=$ liberal, 7 = conservative ; Maertens et al., 2022) to determine criterion validity of the Neural-BFI-20.

Mirroring our analytical approach in STEP 4, we first investigated reliability and structural validity. Regarding the former, for all five dimensions of the N-BFI-20, test-retest correlations consistently exceeded the customary threshold of $r=.70$ that we had preregistered, indicating good (Openness: $r=.747, p<.001$; Agreeableness: $r=.775, p<.001$ ) to excellent test-retest reliability (Conscientiousness: $r=.866, p<.001$; Extraversion: $r$ $=.901, p<.001$; Neuroticism: $r=.884, p<.001)$. Regarding the latter, this novel dataset reproduced the results obtained in the student sample in STEP 4, while improving on all metrics. That is, the N-BFI-20 exhibited excellent fit for the RMSEA $(.044,90 \%$ CI 
$[.033, .054)$, SRMR (0.026), CFI (.97), and TL (.95) criteria (Clark \& Watson, 2019; Finch \& West, 1997; Hu \& Bentler, 1999; Pituch \& Stevens, 2015; Schumacker et al., 2015). As before, the $\chi^{2}$ goodness of fit test reached significance $\left(\chi^{2}=176.47, p<.001\right)$, which may, however, be due to the test's sample size sensitivity (Bentler \& Bonett, 1980; Curran et al., 2003; Rosellini \& Brown, 2021).

Parallel to our analytical approach in STEP 4, subsequently we investigated the associations between the N-BFI-20 dimensions and various practical outcomes across different life domains, to assess predictive validity (Clifton, 2020). To that end, informed by the existing literature we predicted — and pre-registered — the following relationships: H5: Personal health will be positively correlated with Conscientiousness and Extraversion, as well as negatively correlated with Neuroticism (Gosling et al., 2003; Rammstedt et al., 2020).

H6: Life satisfaction will be positively correlated with Conscientiousness, Extraversion, and Agreeableness, as well as negatively correlated with Neuroticism (Rammstedt et al., 2020). H7: Educational attainment will be positively correlated with Openness, and Extraversion, and negatively correlated with Neuroticism (Rammstedt et al., 2020).

H8: Income will be positively correlated with Conscientiousness, and Extraversion, and negatively correlated with Neuroticism (Gosling et al., 2003; Rammstedt et al., 2020). H9: Political liberalism will be positively correlated with Openness, and Neuroticism, and negatively correlated with Conscientiousness (Gosling et al., 2003).

As predicted in H5, Conscientiousness $(r=.309, p<.001)$, and Extraversion $(r$ $=.214, p<.001$ ), were positively correlated with personal health, whereas Neuroticism was negatively correlated with personal health $(r=-.318, p<.001)$.

As predicted in H6, Conscientiousness $(r=.261, p<.001)$, Extraversion $(r=.341, p$ $<.001)$, and Agreeableness $(r=.209, p<.001)$ were positively correlated with life 
satisfaction, whereas Neuroticism was negatively correlated with life satisfaction $(r=-.437, p$ $<.001)$.

As predicted in H7, Neuroticism was negatively correlated with educational attainment $(r=-.140, p=.003)$. However, in opposition with H7, neither Openness $(r=.050$, $p=.295)$, nor Extraversion $(r=.038, p=.421)$, were significantly positively correlated with educational attainment.

As predicted in H8 Conscientiousness $(r=.183, p<.001)$, and Extraversion $(r=.155$, $p=.001$ ), were positively correlated with income, whereas Neuroticism was negatively correlated with income $(r=-.155, p=.001)$.

As predicted in H9 Openness $(r=-.167, p<.001)$, and Neuroticism $(r=-.226, p$ $<.001$ ), were correlated with political liberalism (scale anchored at $1=$ liberal, $7=$ conservative), whereas - contrary to H9-Conscientiousness was not significantly correlated with political conservatism $(r=.084, p=.078)$.

Table 6 contains an overview of internal and external correlations (including 95\% confidence intervals) with significant correlations in bold. Figure 2 provides a visualisation of the complete correlation matrix, summarising all bivariate relationships within the current dataset. 
Table 6

Internal and External Correlation Patterns of the N-BFI-2

\begin{tabular}{|c|c|c|c|c|c|c|}
\hline & & \multicolumn{2}{|c|}{ Pearson's $r$} & \multirow[t]{2}{*}{$p$} & \multicolumn{2}{|c|}{$95 \% \mathrm{CI}$} \\
\hline & & & & & $L L$ & $U \boldsymbol{L}$ \\
\hline \multicolumn{7}{|c|}{ INTERNAL CORRELATIONS } \\
\hline N-BFI-20 T1 O & - $\quad$ N-BFI-20 T2 $\overline{\mathrm{O}}$ & 0.747 & $* * *$ & $<.001$ & 0.703 & 0.785 \\
\hline N-BFI-20 T1 C & - N-BFI-20 T2 C & 0.866 & $* * *$ & $<.001$ & 0.841 & 0.888 \\
\hline N-BFI-20 T1 E & - N-BFI-20 T2 E & 0.901 & $* * *$ & $<.001$ & 0.883 & 0.917 \\
\hline N-BFI-20 T1 A & - N-BFI-20 T2 A & 0.775 & $* * *$ & $<.001$ & 0.735 & 0.810 \\
\hline N-BFI-20 T1 N & - $\quad$ N-BFI-20 T2 N & 0.884 & $* * *$ & $<.001$ & 0.862 & 0.903 \\
\hline N-BFI-20 T2 O & - N-BFI-20 T2 C & 0.254 & $* * *$ & $<.001$ & 0.165 & 0.339 \\
\hline N-BFI-20 T2 O & - N-BFI-20 T2 E & 0.297 & $* * *$ & $<.001$ & 0.210 & 0.379 \\
\hline N-BFI-20 T2 O & - $\quad$ N-BFI-20 T2 A & 0.303 & $* * *$ & $<.001$ & 0.216 & 0.385 \\
\hline N-BFI-20 T2 O & - $\quad$ N-BFI-20 T2 N & -0.240 & $* * *$ & $<.001$ & -0.325 & -0.151 \\
\hline N-BFI-20 T2 C & - N-BFI-20 T2 E & 0.232 & $* * *$ & $<.001$ & 0.143 & 0.318 \\
\hline N-BFI-20 T2 C & - $\quad$ N-BFI-20 T2 A & 0.237 & $* * *$ & $<.001$ & 0.148 & 0.322 \\
\hline N-BFI-20 T2 C & - N-BFI-20 T2 N & -0.269 & $* * *$ & $<.001$ & -0.353 & -0.181 \\
\hline N-BFI-20 T2 E & - $\quad$ N-BFI-20 T2 A & 0.243 & $* * *$ & $<.001$ & 0.154 & 0.328 \\
\hline N-BFI-20 T2 E & - N-BFI-20 T2 N & -0.315 & $* * *$ & $<.001$ & -0.396 & -0.230 \\
\hline N-BFI-20 T2 A & - N-BFI-20 T2 N & -0.196 & $* * *$ & $<.001$ & -0.284 & -0.106 \\
\hline \multicolumn{7}{|c|}{ EXTERNAL CORRELATIONS } \\
\hline N-BFI-20 T2 O & - Age & 0.043 & & 0.368 & -0.050 & 0.135 \\
\hline N-BFI-20 T2 O & - Gender: Male & -0.063 & & 0.191 & -0.156 & 0.031 \\
\hline N-BFI-20 T2 O & - Education & 0.050 & & 0.295 & -0.043 & 0.142 \\
\hline N-BFI-20 T2 O & - Ideology & -0.167 & $* * *$ & $<.001$ & -0.256 & -0.076 \\
\hline N-BFI-20 T2 O & - Income & 0.000 & & 0.992 & -0.094 & 0.093 \\
\hline N-BFI-20 T2 O & - Health & 0.152 & $* *$ & 0.001 & 0.060 & 0.241 \\
\hline N-BFI-20 T2 O & - Life Satisfaction & 0.176 & $* * *$ & $<.001$ & 0.084 & 0.264 \\
\hline N-BFI-20 T2 C & - Age & 0.071 & & 0.133 & -0.022 & 0.163 \\
\hline N-BFI-20 T2 C & - Gender: Male & 0.045 & & 0.348 & -0.049 & 0.139 \\
\hline N-BFI-20 T2 C & - Education & 0.127 & $* *$ & 0.007 & $\mathbf{0 . 0 3 4}$ & 0.217 \\
\hline N-BFI-20 T2 C & - Ideology & 0.084 & & 0.078 & -0.009 & 0.175 \\
\hline N-BFI-20 T2 C & - Income & 0.183 & $* * *$ & $<.001$ & 0.091 & 0.272 \\
\hline N-BFI-20 T2 C & - Health & 0.309 & $* * *$ & $<.001$ & 0.223 & 0.391 \\
\hline N-BFI-20 T2 C & - $\quad$ Life Satisfaction & 0.261 & $* * *$ & $<.001$ & 0.173 & 0.346 \\
\hline N-BFI-20 T2 E & - Age & 0.058 & & 0.219 & -0.035 & 0.150 \\
\hline N-BFI-20 T2 E & - Gender: Male & -0.057 & & 0.239 & -0.150 & 0.038 \\
\hline N-BFI-20 T2 E & - Education & 0.038 & & 0.421 & -0.055 & 0.130 \\
\hline N-BFI-20 T2 E & - Ideology & 0.065 & & 0.172 & -0.028 & 0.157 \\
\hline N-BFI-20 T2 E & - Income & 0.155 & $* *$ & 0.001 & 0.062 & 0.245 \\
\hline N-BFI-20 T2 E & - Health & 0.214 & $* * *$ & $<.001$ & 0.124 & 0.301 \\
\hline N-BFI-20 T2 E & - Life Satisfaction & 0.341 & $* * *$ & $<.001$ & 0.256 & 0.420 \\
\hline N-BFI-20 T2 A & - Age & 0.028 & & 0.548 & -0.064 & 0.121 \\
\hline N-BFI-20 T2 A & - Gender: Male & -0.148 & $* *$ & 0.002 & -0.239 & -0.055 \\
\hline N-BFI-20 T2 A & - Education & -0.009 & & 0.854 & -0.101 & 0.084 \\
\hline N-BFI-20 T2 A & - Ideology & -0.130 & $* *$ & 0.006 & -0.220 & -0.037 \\
\hline N-BFI-20 T2 A & - Income & 0.047 & & 0.327 & -0.047 & 0.140 \\
\hline
\end{tabular}




\begin{tabular}{|c|c|c|c|c|c|c|}
\hline N-BFI-20 T2 A & - Health & 0.176 & $* * *$ & $<.001$ & 0.085 & 0.264 \\
\hline N-BFI-20 T2 A & - Life Satisfaction & 0.211 & $* * *$ & $<.001$ & 0.121 & 0.298 \\
\hline N-BFI-20 T2 N & - Age & -0.175 & $* * *$ & $<.001$ & -0.263 & -0.084 \\
\hline N-BFI-20 T2 N & - Gender: Male & -0.280 & $* * *$ & $<.001$ & -0.365 & -0.191 \\
\hline N-BFI-20 T2 N & - Education & -0.140 & $* *$ & 0.003 & -0.230 & -0.048 \\
\hline N-BFI-20 T2 N & - Ideology & -0.226 & $* * *$ & $<.001$ & -0.312 & -0.136 \\
\hline N-BFI-20 T2 N & - Income & -0.155 & $* *$ & 0.001 & -0.245 & -0.062 \\
\hline N-BFI-20 T2 N & - Health & -0.318 & $* * *$ & $<.001$ & -0.399 & -0.232 \\
\hline N-BFI-20 T2 N & - $\quad$ Life Satisfaction & -0.437 & $* * *$ & $<.001$ & -0.510 & -0.359 \\
\hline
\end{tabular}

Note. Bold cells represent significant correlations. $N=451 . \mathrm{O}=$ Openness. $\mathrm{C}=$ Conscientiousness. $\mathrm{E}=$

Extraversion. $\mathrm{A}=$ Agreeableness. $\mathrm{N}=$ Neuroticism. $\mathrm{CI}=$ confidence interval. $L L=$ lower level. $U L=$ upper level. $* * * p<.001, * * p<.01, * p<.05$

\section{Figure 2}

Bivariate Correlation Matrix_General Canadian Adult Population Sample

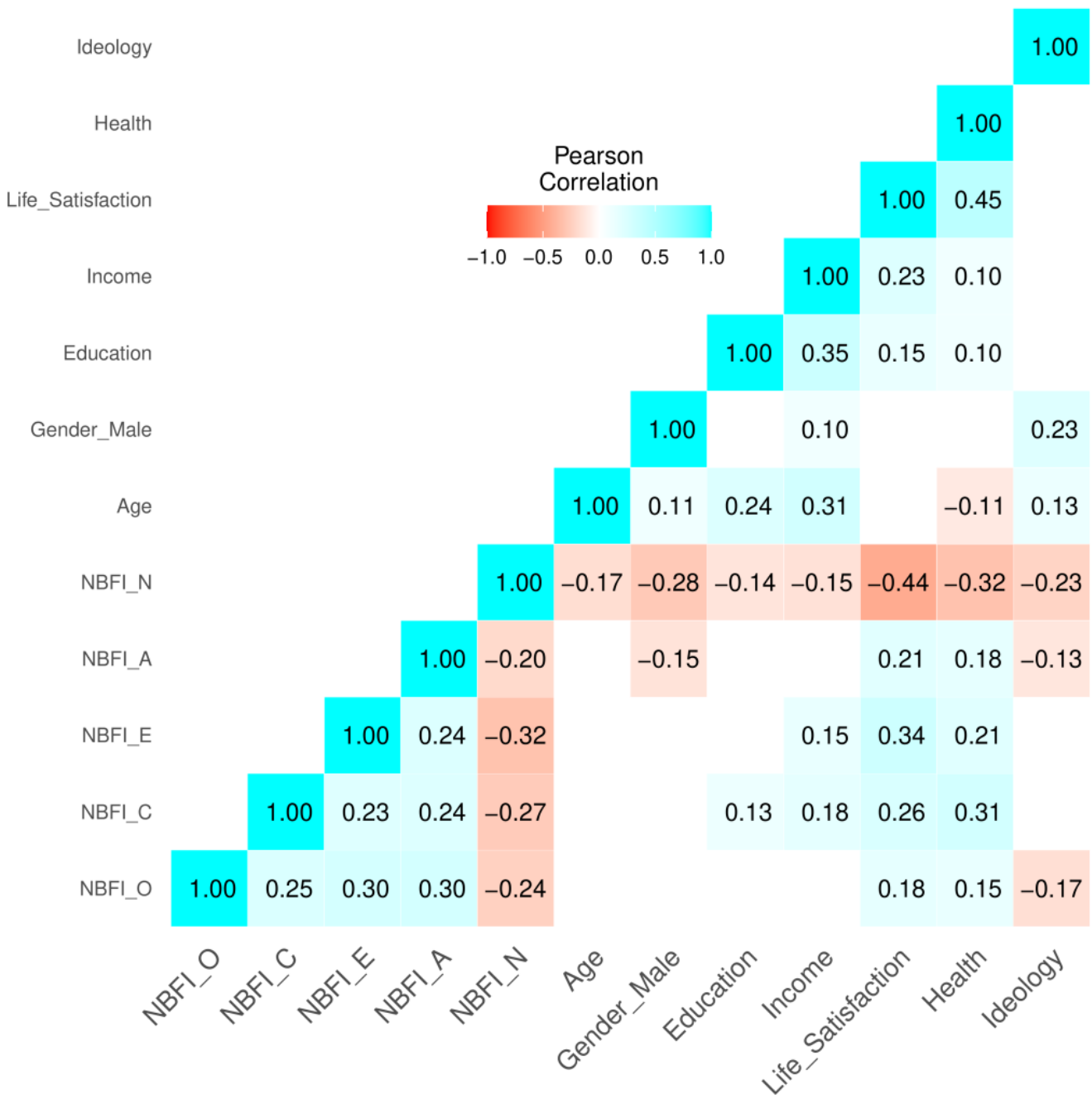

\section{Summary}


Across five pre-registered steps, we illustrated the applied value of the PIG for psychometric development by designing and empirically validating a novel Big Five short scale, the N-BFI-20. In STEP 1 we fed the BFI-2 (Soto \& John, 2017), the current gold standard of Big Five measurement, into the PIG and parsed it into 10 input iterations (each one regular- and reverse-keyed per Big Five dimension), to generate a large initial item pool, which encompassed several thousand items. In STEP 2 we set up a culturally-diverse doubleblind expert committee to pre-select a more manageable set of items for empirical testing, based on conceptual relevance, linguistic clarity, and balanced keying. This resulted in a selection of 92 unedited items, 89 of which had been unanimously chosen. In STEP 3 we recruited a sample of the general Canadian population $(N=501)$ via Prolific, and administered the pre-selected item pool and demographic questions. We then employed a combination of factor analyses and item response theory to extract the best-performing set of 20 items (four per personality dimension, thereof each two regular- and reverse-keyed) which make up the N-BFI-20. In STEP 4 we recruited a novel sample of university students from the University of British Columbia $(N=773)$, who completed the N-BFI-20, the BFI-2, a battery of sociodemographic questions and measures designed to assess social dominance orientation, music preferences, and self-esteem. We then first conducted basic psychometric analyses to gauge internal consistency (via McDonald's $\omega$ ) and structural validity (via confirmatory factor analyses and exploratory structural equation modelling) and subsequently simultaneously investigated correlation patterns of the N-BFI-20 and BFI-2, both with each other and with the remaining constructs. This direct head-to-head comparison offered the dual advantage of enabling us to evaluate the performance of the N-BFI-20 both, in absolute terms (i.e., through established psychometric indices and through inspecting its pre-registered nomological net) but also relative to a well-established, traditionally-generated Big Five measure (Flake et al., 2017). In STEP 5 we revisited the Prolific sample from STEP 3 to 
consolidate and extend the analyses undertaken in STEP 4. In addition to replicating the exploratory structural equation modelling analyses to probe structural validity, we assessed test-retest reliability $(N=451 ; M$ interval $=24$ days $)$ and related the N-BFI-20 to diverse realworld outcomes (i.e., personal health, life satisfaction, educational attainment, income and political orientation) to gauge its predictive validity. Overall, the empirical investigations provided ample support for the utility of the N-BFI-20, both as a parsimonious measure of the Big Five and vis-à-vis the BFI-2 as the current state-of-the-art in Big Five assessment. The N-BFI-20 consistently accomplished satisfactory reliability estimates, across different samples and both in terms of internal consistency (i.e., McDonald's $\omega$ ) and test-rest reliability. Likewise, across both samples, ESEM revealed good to excellent structural validity for the N-BFI-20. Encouragingly, investigating the N-BFI-20's nomological network both independent of and in relation to the BFI-2 provided strong evidence for the construct validity of the scale. Of 13 pre-registered associations within the N-BFI-20's nomological network in STEP 4, 7 (i.e., 53.8\%) were empirically supported, with those that were not almost always exhibiting coefficients aligned with the expected effect direction. Furthermore, the N-BFI-20 consistently not only showed the same predicted relationship directions as the BFI-2, but typically also exhibited effect sizes of near-same or very similar magnitude. Importantly, whenever the N-BFI-20 deviated from the predicted association, the exact same pattern was found for the BFI-2. As our predictions were based on previous validation of other Big Five measures that had either been carried out almost two decades ago (Gosling et al., 2003) or primarily relied on culturally different samples (e.g., Germans; Rammstedt et al., 2020), it is highly plausible that these instances reflect a genuine signal, rather than a lack of construct validity. For example, it appears reasonable to assume that whereas in the early 2000s (i.e., when Gosling et al., 2003, conducted their study), Conscientiousness and Agreeableness were related to a preference for upbeat and conventional music, given the 
cultural shifts that have occurred since, this may no longer be the case (if it has ever been true for Canadians). Further lending credibility to the construct validity of the N-BFI-20, when juxtaposed with the BFI-2, not only were identically named dimensions consistently most highly correlated across measures (e.g., N-BFI-20 Openness and BFI-2 Openness), but the NBFI-20 also reproduced the inter-factor correlation patterns found in the BFI-2 (see Table 5 and Figure 1). Similarly, STEP 5 compiled clear evidence for the predictive validity of the N-BFI-20, with 12 of 15 pre-registered associations with important life outcomes (i.e., 80\%) receiving empirical support.

Taken together, when tested in the wild, the PIG-generated N-BFI-20 revealed itself to be a potent and parsimonious Big Five scale, with impressive psychometric properties and predictive power for diverse real-world outcomes that performs as well as the rigorouslydeveloped, widely-established BFI-2 - at only one third of its length. It is important to note that the current validation is not just a theoretical exercise. Indeed, in times of widespread utilization of crowd-working platforms in psychological science (Gosling \& Mason, 2015; Paolacci \& Chandler, 2014), which leave large groups of researchers to conduct research on a limited group of individuals (Hauser et al., 2019; Stewart et al., 2015), hence raising concerns about prior exposure and nonnaïvité (Buhrmester et al., 2018; Chandler et al., 2014), there is a real, and growing demand for valid short scales and parallel forms. With this in mind, we believe that there is a bright future for scales such as the N-BFI-20, which—as the current test case demonstrates - may be created by algorithms but are fit for usage among humans.

\section{General Discussion}

Psychological scientists across the discipline rely on well-designed measures to assess latent constructs (Boateng et al., 2018; Hinkin, 1995; Simms, 2008). To meet the resultant demand, sound scale development is key (Morgado et al., 2017; Sellbom \& Simms, 2019; Rosellini \& Brown, 2021). Traditionally, an equally essential and effortful part of scale 
development regards the manual generation of large numbers of high-quality items (Clark \& Watson, 1995, 2019; Loevinger, 1957; Worthington \& Whittaker, 2006). To turn item production from an art (Zickar, 2020) to automation, the current tutorial draws from recent breakthroughs in data science and artificial intelligence (Radford et al., 2019a,b) to pave the way for flexible, easily implementable, large-scale item generation for psychological measurement. To that end, we provide a fully functional digital infrastructure that harnesses an extremely powerful natural language processing machine learning algorithm, runs on state-of-the-art hardware, and enables psychologists to generate massive amounts of coherent, customised items within mere minutes, at zero costs and without requiring any coding knowledge, software packages, or computational resources. We then showcase the utility of our tool — the Psychometric Item Generator (PIG) — across two applied test cases, generating a face-valid large candidate item pool for a completely unstudied construct (i.e., wanderlust) and developing and empirically validating a short scale of the most prominent personality taxonomy (i.e., the Big Five) which hits the mark when benchmarked against the gold standard in the field as well as when evaluated on its own.

\section{Limitations}

Of course, the current approach is not without limitations. Just like one should not blindly rely on human intelligence, the same is true for artificial intelligence. Imperfect algorithmic performance can and does occur (Radford et al., 2019a). While repeated iterations and parameter tweaking (e.g., by adjusting top_p and temperature to increase or decrease linguistic diversity) can often offer quick relief, some PIG executions may yield less useful items than others. Importantly, no form of automated item generation currently available fully obviates the need for formal quality checks and human review (Goldenfein, 2019; Köbis \& Mossink, 2021; Royal et al., 2018). That is, even within the best iterations, most of what the PIG — or any other comparable algorithm—generates will not be usable and 
the onus is one the researcher to read, assess, and organise the extensive algorithmic output to select suitable items. To illustrate — not considering the occasional unstructured text output that does not conform to the modelled item format — out of the 294 items that the single wanderlust iteration of the PIG produced, 65 items — or about one fourth—passed an initial face validity check to be added to a larger item pool for the development of a wanderlust scale (see Demonstration 1 above and OM1 for complete output). Thus, while we believe that there are significant benefits to using the PIG as outlined above (e.g., scalability, broader and more diverse conceptualisations that move beyond the restrictions of any individual researcher's or research team's understanding of a construct, useful cues to potential relationships with other constructs), both traditional, manual item generation and the modern, automated item generation presented here come with their own unique trade-offs. Hence, it is ultimately a matter of research goals, context and researcher preferences which approach will serve better. In the end, regardless of how they are constructed, algorithmically generated items and their human-written counterparts have to undergo the same rigorous field-testing procedures and subsequent empirical evaluation (Gierl et al., 2012). As such, we like to think of the current work as extending — rather than replacing — existing approaches to item development just as we conceive of the PIG as complementing — rather than supplementingmanual item generation.

Secondly, the current version of the PIG is based on the generic, pre-trained GPT-2. While fine-tuning of the $1558 \mathrm{M}$ and $774 \mathrm{M}$ GPT-2 model—which the current PIG is usingis possible, this falls outside the scope of this tutorial. Future applications may yield even better results by re-training the GPT-2 on custom-tailored text corpora, such as psychological scale databases. Indeed, first examples of targeted fine-tuning are already producing encouraging initial results and foreshadow the minute task-specific calibration that may be accomplished in the future (Hommel et al., 2021; Howard \& Ruder, 2018; von Davier, 
2019)—while other scholars suggest that the ever-increasing powers of coming generations of generative language models will soon remove the need and incentive for re-training as peak performance can be accomplished without requiring any further fine-tuning (Han et al., 2021; Launay et al., 2021; Yang, 2021).

Lastly, as with any free platform, a risk of using Google Colab to host and power the PIG is that it might turn into a paid service or be discontinued (Nelson \& Hoover, 2020). While the Jupyter notebook that runs the PIG is not strictly dependent on Colab and can be run on other platforms or locally downloaded as a python script (von Chamier et al., 2021), if Colab were to stop operating, users would need to find other ways to access adequate computational power to execute the notebooks. Fortunately, many free alternatives to Google Colab exist, among others, Paperspace Gradient (https://gradient.paperspace.com/), Kaggle (https://www.kaggle.com/), Amazon SageMaker (https://aws.amazon.com/sagemaker/), and FloydHub (https://www.floydhub.com/).

\section{Outlook and Conclusions}

We believe that the PIG has a bright future and are convinced that it is capable of many more things than we have been able to show here. Future applications may include dictionary development (Eichstaedt et al., 2021; Kennedy et al., 2021), wherein users could feed the PIG single construct-relevant words and harness the deep neural networks to collect related words. Yet others may want to enlist the PIG's help in writing vignettes. More exciting still, future research may create and use extensions of the PIG to drive automated item generation in non-English languages. Indeed, first transfer learning approaches retraining the GPT-2 to Dutch, Italian (de Vries et al., 2020), and Portuguese (Guilllou, 2020) have shown promising results, as have original GPT-type architectures that were trained from scratch using large-scale text corpora in other languages, such as Arabic (AraGPT2; Antoun et al., 2020), Chinese (Pangu; Zeng et al., 2021; CPM; Zhang et al., 2021) French (PAGnol; 
Launay et al., 2021), Romanian (RoGPT-2; Niculescu et al., 2021) and Russian (ruGPT3; Alexandr \& Arina, 2021). Importantly, such approaches may not be limited to national languages. Initial evidence shows that targeted retraining of the GPT-2 may enable users to generate authentic regional or socio-historical language variants, such as Swiss-German (Linder et al., 2020), Arabic dialects (Doiron, 2020), or Shakespearean English (Chhabra, 2020). As such approaches mature and become more widely implementable, they may pave the way for new forms of cross-cultural psychological assessment which go beyond direct back-translation procedures (Behling \& Law, 2000; Brislin, 1970; Moshontz et al., 2018) and might pioneer AI-driven combined emic-etic approaches to scale development (BenetMartínez, 1999; Cheung et al., 2011; Yang \& Bond, 1990) that attend to the many layers of culture (Cohen, 2009). Taken together it is easy to imagine a number of ways in which the PIG can be used to advance psychological measurement and research. However, the powers of the GPT-2 and the PIG can also be abused to create fake news, impersonate others online, or produce spam and phishing content (Radford et al., 2019a). Thankfully, as of yet there is little of evidence of the prevalence of these practices. Nonetheless, as advanced computer technologies are rapidly entering mainstream research, psychologists must—and increasingly do-discuss the ethical challenges and personal responsibilities that arise from using these technologies (Alexander et al., 2020; Kosinski et al., 2015, 2016; Matz et al., 2020), including powerful language algorithms like the GPT-2 and their applications like the PIG. Building equally powerful detector models designed to monitor output and flag up misuse and releasing them alongside the primary generative models, as modelled by Antoun et al. (2020), may be one effective way to tackle this issue as the field evolves.

We would like to end on a note of humility and gratitude. Artificial intelligence is an extremely dynamic and fast-growing field. As a case in point, OpenAI released the GPT-3 (Brown et al., 2021) as we were writing this tutorial. We thus have no illusions that the PIG 
as presented here will be outperformed sooner rather than later; nor do we wish to take any credit for the amazing achievements of data scientists and AI researchers that freely share their resources and are the true giants upon whose shoulders applications like the PIG can come into being. Instead, we understand our contribution as one that is first and foremost translational, with the aim of bringing powerful new AI tools to a wider psychological audience. In other words, we sought to introduce, explain, and apply the PIG in a language that is familiar so that you in turn can let the algorithm speak. 


\section{References}

Abadi, M., Agarwal, A., Barham, P., Brevado, E., Chen, Z., Citro, C., ... Zheng, X. (2016). TensorFlow: Large-scale machine learning on heterogeneous distributed systems. arXiv. https://doi.org/10.48550/arXiv.1605.08695

Ackley, D. H., Hinton, G. E., \& Sejnowski, T. J. (1985). A learning algorithm for boltzmann machines. Cognitive Science, 9 (1), 147-16.https://doi.org/10.1016/S03640213(85)80012-4

Alexander, L., Mulfinger, E., \& Oswald, F. L. (2020). Using big data and machine learning in personality measurement: Opportunities and challenges. European Journal of Personality, 34(5), 632-648. https://doi.org/10.1002/per.2305

Alexandr N., Irina O., Tatyana K., Inessa K., Arina P. (2021) Fine-tuning GPT-3 for Russian text summarization. In: Silhavy R., Silhavy P., Prokopova Z. (Eds.), Data science and intelligent systems. CoMeSySo 2021. Lecture notes in networks and systems (pp. 748757). Springer. https://doi.org/10.1007/978-3-030-90321-3_61

Anastasi, A., \& Urbina, S. (1997). Psychological testing. Prentice Hall.

Antoun, W., Baly, F., \& Hajj, H. (2020). AraGPT2: Pre-trained transformer for Arabic language generation. arXiv preprint, arXiv:2012.15520.

Arendasy, M., \& Sommer, M. (2005). The effect of different types of perceptual manipulations on the dimensionality of automatically generated figural matrices. Intelligence, 33(3), 307-324. https://doi.org/10.1016/j.intell.2005.02.002

Arendasy, M., \& Sommer, M. (2007). Using psychometric technology in educational assessment: The case of a schema-based isomorphic approach to the automatic generation of quantitative reasoning items. Learning and Individual Differences, 17(4), 366-383. https://doi.org/10.1016/j.lindif.2007.03.005 
Arendasy, M. E., \& Sommer, M. (2010). Evaluating the contribution of different item features to the effect size of the gender difference in three-dimensional mental rotation using automatic item generation. Intelligence, 38(6), 574-581. https://doi.org/10.1016/j.intell.2010.06.004

Arendasy, M. E., \& Sommer, M. (2012). Using automatic item generation to meet the increasing item demands of high-stakes educational and occupational assessment. Learning and Individual Differences, 22(1), 112-117. https://doi.org/10.1016/j.lindif.2011.11.005

Asparouhov, T., \& Muthén, B. (2009). Exploratory structural equation modeling. Structural Equation Modeling: A Multidisciplinary Journal, 16(3), 397-438. https://doi.org/10.1080/10705510903008204

Bahdanau, D., Cho, K., \& Bengio, Y. (2016). Neural machine translation by jointly learning to align and translate. arXiv preprint, arXiv:1409.0473v7.

Baumeister, R. F., Vohs, K. D., \& Funder, D. C. (2007). Psychology as the science of selfreports and finger movements: Whatever happened to actual behavior? Perspectives on Psychological Science, 2(4), 396-403. https://doi.org/10.1111/j.17456916.2007.00051.x

Behling, O., \& Law, K. S. (2000). Translating questionnaires and other research instruments: Problems and solutions. SAGE Publications, Inc. https://doi.org/10.4135/9781412986373

Beierlein, C., Kovaleva, A., László, Z., Kemper, C. J., \& Rammstedt, B. (2014). Eine SingleItem-Skala zur Erfassung der Allgemeinen Lebenszufriedenheit: Die Kurzskala Lebenszufriedenheit-1 (L-1) [A single-item scale measuring general life satisfaction]. Köln, Germany: GESIS. (GESIS Working Papers 2014, 33). https://www.gesis.org/fileadmin/kurzskalen/working_papers/L1_WorkingPapers_2014- 
33.pdf

Benet-Martínez, V. (1999). Exploring indigenous Spanish personality constructs with a combined emic-etic approach. In J. C. Lasry, J. G. Adair, \& K. L. Dion, (Eds.), Latest contributions to cross-cultural psychology (pp. 151-175). Routledge. https://doi.org/10.1201/9781003077466-13

Bengio, Y., Ducharme, R., Vincent, P., \& Janvin, C. (2003). A neural probabilistic language model. Journal of Machine Learning Research, 3, 1137-1155.

Bentler, P. M., \& Bonett, D. G. (1980). Significance tests and goodness of fit in the analysis of covariance structures. Psychological Bulletin, 88(3), 588-606. https://doi.org/10.1037/0033-2909.88.3.588

Bisong, E. (2019). Google Colaboratory. In E. O. Bisong (Ed.), Building machine learning and deep learning models on Google Cloud Platform (pp. 59-64). Apress. https://doi.org/10.1007/978-1-4842-4470-8_7

Boateng, G. O., Neilands, T. B., Frongillo, E. A., Melgar-Quiñonez, H. R., \& Young, S. L. (2018). Best practices for developing and validating scales for health, social, and behavioral research: A primer. Frontiers in Public Health, 6. https://doi.org/10.3389/fpubh.2018.00149

Bock, R. D., Gibbons, R., \& Muraki, E. (1988). Full-information item factor analysis. Applied Psychological Measurement, 12, 261-280. http://dx.doi.org/10.1177/014662168801200305

Borkenau, P., \& Ostendorf, F. (1990). Comparing exploratory and confirmatory factor analysis: A study on the 5-factor model of personality. Personality and Individual Differences, 11(5), 515-524. https://doi.org/10.1016/0191-8869(90)90065-y

Brislin, R. W. (1970). Back-translation for cross-cultural research. Journal of Cross-Cultural Psychology, 1(3), 185-216. https://doi.org/10.1177/135910457000100301 
Buhrmester, M. D., Talaifar, S., \& Gosling, S. D. (2018). An evaluation of Amazon's Mechanical Turk, its rapid rise, and its effective use. Perspectives on Psychological Science, 13(2), 149-154. https://doi.org/10.1177/1745691617706516

Chandler, J., Mueller, P., \& Paolacci, G. (2014). Nonnaïveté among Amazon Mechanical Turk workers: Consequences and solutions for behavioral researchers. Behavior Research Methods, 46(1), 112-130. https://doi.org/10.3758/s13428-013-0365-7

Cheung, F. M., van de Vijver, F. J. R., \& Leong, F. T. L. (2011). Toward a new approach to the study of personality in culture. American Psychologist, 66(7), 593-603. https://doi.org/10.1037/a0022389

Chhabra, P. (2020). Using GPT-2 to write like Shakespeare. ABCOM Education. http://education.abcom.com/using-gpt-2-to-write-like-shakespeare/

Chiorri, C., Marsh, H. W., Ubbiali, A., \& Donati, D. (2016). Testing the factor structure and measurement invariance across gender of the Big Five inventory through exploratory structural equation modeling. Journal of Personality Assessment, 98(1), 88-99. https://doi.org/10.1080/00223891.2015.1035381

Church, A. T., \& Burke, P. J. (1994). Exploratory and confirmatory tests of the Big Five and Tellegen's three- and four-dimensional models. Journal of Personality and Social Psychology, 66, 93-114. https://doi.org/10.1037/0022-3514.66.1.93

Clark, L. A., \& Watson, D. (1995). Constructing validity: Basic issues in objective scale development. Psychological Assessment, 7(3), 309-319. https://doi.org/10.1037/10403590.7.3.309

Clark, L. A., \& Watson, D. (2019). Constructing validity: New developments in creating objective measuring instruments. Psychological Assessment, 31(12), 1412-1427. https://doi.org/10.1037/pas0000626 
Clifton, J. D. W. (2020). Managing validity versus reliability trade-offs in scale-building decisions. Psychological Methods, 25, 259-270. https://doi.org/10.1037/met0000236

Cohen, A. B. (2009). Many forms of culture. American Psychologist, 64(3), 194-204. https://doi.org/10.1037/a0015308

Comrey, A. L. (1988). Factor-analytic methods of scale development in personality and clinical psychology. Journal of Consulting and Clinical Psychology, 56(5), 754-761. https://doi.org/10.1037/0022-006x.56.5.754

Costello, A. B., \& Osborne, J. (2005). Best practices in exploratory factor analysis: Four recommendations for getting the most from your analysis. Practical Assessment, Research, and Evaluation, 10. https://doi.org/10.7275/jyj1-4868

Cronbach, L. J. (1946). Response sets and test validity. Educational and Psychological Measurement, 6, 475-494. https://doi.org/10.1177/001316444600600405

Curran, P. J., Bollen, K. A., Chen, F., Paxton, P., \& Kirby, J. B. (2003). Finite sampling properties of the point estimates and confidence intervals of the RMSEA. Sociological Methods \& Research, 32, 208-252. https://doi.org/10.1177/0049124103256130

Danner, D., Aichholzer, J., \& Rammstedt, B. (2015). Acquiescence in personality questionnaires: Relevance, domain specificity, and stability. Journal of Research in Personality, 57, 119-130. https://doi.org/10.1016/j.jrp.2015.05.004

de Vries, W., \& Nissim, M. (2020). As good as new: How to successfully recycle English GPT-2 to make models for other languages. ArXiv. https://arxiv.org/abs/2012.05628

DeVellis, R. F. (2003). Scale development: Theory and applications. Sage.

DeVellis, R. F. (2016). Scale development: Theory and applications (4th ed.). Sage. https://us.sagepub.com/en-us/nam/scale-development/book246123

Devlin, J., Chang, M.-W., Lee, K., \& Toutanova, K. (2019). BERT: Pre-training of deep bidirectional transformers for language understanding. In Proceedings of the 2019 
Conference of the North American Chapter of the Association for Computational

Linguistics: Human Language Technologies, Volume 1 (Long and Short Papers) (pp. 4171-4186). https://doi.org/10.18653/v1/N19-1423

Doiron, N. (2020). Making a mini GPT-2 with dialect prompts. Medium. https://mapmeld.medium.com/making-a-mini-gpt-2-with-dialect-prompts$605 \mathrm{a} 0 \mathrm{edbc} 1 \mathrm{e} 4$

Donahue, J., Anne Hendricks, L., Guadarrama, S., Rohrbach, M., Venugopalan, S., Saenko, K., \& Darrell, T. (2015). Long-term recurrent convolutional networks for visual recognition and description. In Proceedings of the IEEE conference on computer vision and pattern recognition (pp. 2625-2634).

Donnellan, M. B., Oswald, F. L., Baird, B. M., \& Lucas, R. E. (2006). The Mini-IPIP scales: Tiny-yet-effective measures of the Big Five factors of personality. Psychological Assessment, 18(2), 192-203. https://doi.org/10.1037/1040-3590.18.2.192

Dunn, T. J., Baguley, T., \& Brunsden, V. (2014). From alpha to omega: A practical solution to the pervasive problem of internal consistency estimation. British Journal of Psychology, 105(3), 399-412. https://doi.org/10.1111/bjop.12046

Eichstaedt, J. C., Kern, M. L., Yaden, D. B., Schwartz, H. A., Giorgi, S., Park, G., Hagan, C. A., Tobolsky, V. A., Smith, L. K., Buffone, A., Iwry, J., Seligman, M. E. P., \& Ungar, L. H. (2021). Closed- and open-vocabulary approaches to text analysis: A review, quantitative comparison, and recommendations. Psychological Methods, 26(4), 398427. https://doi.org/10.1037/met0000349

Elman, J. L. (1990). Finding structure in time. Cognitive Science, 14 (2), 179-211. https://doi.org/10.1207/s15516709cog1402\_1 
Embretson, S. E., \& Yang, X. (2007). Automatic item generation and cognitive psychology. In C. R. Rao \& S. Sinharay (Eds.), Handbook of statistics: Psychometrics (Vol. 26, pp. 747-768). Elsevier. https://doi.org/10.1016/S0169-7161(06)26023-1

Fabrigar, L. R., Wegener, D. T., MacCallum, R. C., \& Strahan, E. J. (1999). Evaluating the use of exploratory factor analysis in psychological research. Psychological Methods, 4(3), 272-299. https://doi.org/10.1037/1082-989X.4.3.272

Fan, A., Lewis, M., \& Dauphin, Y. (2018). Hierarchical neural story generation. ArXiv. https://arxiv.org/abs/1805.04833

Faul, F., Erdfelder, E., Buchner, A., \& Lang, A. G. (2009). Statistical power analyses using G*Power 3.1: Tests for correlation and regression analyses. Behavior Research Methods, 41, 1149-1160. https://doi.org/10.3758/brm.41.4.1149

Faul, F., Erdfelder, E., Lang, A. G., \& Buchner, A. (2007). G*Power 3: A flexible statistical power analysis program for the social, behavioral, and biomedical sciences. Behavior Research Methods, 39, 175-191. https://doi.org/10.3758/bf03193146

Fetaya, E., Lifshitz, Y., Aaron, E., \& Gordin, S. (2020). Restoration of fragmentary Babylonian texts using recurrent neural networks. Proceedings of the National Academy of Sciences, 117(37), 22743-22751. https://doi.org/10.1073/pnas.2003794117

Finch, J. F., \& West, S. G. (1997). The investigation of personality structure: Statistical models. Journal of Research in Personality, 31(4), 439-485. https://doi.org/10.1006/jrpe.1997.2194

Flake, J. K., Pek, J., \& Hehman, E. (2017). Construct validation in social and personality research. Social Psychological and Personality Science, 8(4), 370-378. https://doi.org/10.1177/1948550617693063

Ford, J. K., MacCallum, R. C., \& Tait, M. (1986). The application of exploratory factor analysis in applied psychology: A critical review and analysis. Personnel Psychology, 
39(2), 291-314. https://doi.org/10.1111/j.1744-6570.1986.tb00583.x

Funder, D. C., \& Ozer, D. J. (2019). Evaluating effect size in psychological research: Sense and nonsense. Advances in Methods and Practices in Psychological Science, 2(2), 156168. https://doi.org/10.1177/2515245919847202

Ganegedara, T. (2018). Natural Language Processing with TensorFlow: Teach language to machines using Python's deep learning library. Packt Publishing Ltd.

Gehlbach, H., \& Brinkworth, M. E. (2011). Measure twice, cut down error: A process for enhancing the validity of survey scales. Review of General Psychology, 15(4), 380387. https://doi.org/10.1037/a0025704

Ghiselli, E. E., Campbell, J. P., \& Zedeck, S. (1981). Measurement theory for the behavioral sciences. WH Freeman.

Gierl, M. J., \& Haladyna, T. M. (2012). Automatic item generation: Theory and practice. Routledge. https://doi.org/10.4324/9780203803912

Gierl, M. J., \& Lai, H. (2013). Evaluating the quality of medical multiple-choice items created with automated processes. Medical Education, 47(7), 726-733. https://doi.org/10.1111/medu.12202

Gierl, M. J., Lai, H., \& Turner, S. R. (2012). Using automatic item generation to create multiple-choice test items. Medical Education, 46(8), 757-765. https://doi.org/10.1111/j.1365-2923.2012.04289.x

Gierl, M. J., Lai, H., Pugh, D., Touchie, C., Boulais, A. P., \& De Champlain, A. (2016). Evaluating the psychometric characteristics of generated multiple-choice test items. Applied Measurement in Education, 29(3), 196-210. https://doi.org/10.1080/08957347.2016.1171768

Gignac, G. E., \& Szodorai, E. T. (2016). Effect size guidelines for individual differences researchers. Personality and Individual Differences, 102, 74-78. 
https://doi.org/10.1016/j.paid.2016.06.069

Goldberg, Y. (2017). Neural network methods for natural language processing. Morgan \& Claypool.

Goldenfein, J. (2019). Algorithmic transparency and decision-making accountability: Thoughts for buying machine learning algorithms. In Closer to the machine: Technical, social, and legal aspects of $A I$ (pp. 41-145). https://ssrn.com/abstract=3445873.

Gosling, S. D., \& Mason, W. (2015). Internet research in psychology. Annual Review of Psychology, 66(1), 877-902. https://doi.org/10.1146/annurev-psych-010814-015321

Gosling, S. D., Rentfrow, P. J., \& Swann, W. B. (2003). A very brief measure of the Big-Five personality domains. Journal of Research in Personality, 37(6), 504-528. https://doi.org/10.1016/s0092-6566(03)00046-1

Götz, F. M., Maertens, R., Loomba, S., \& van der Linden S. (2022). Let the algorithm speak: How to use neural networks for automatic item generation in psychological scale development - online materials. https://osf.io/fhpv6/

Guillou, P. (2020). Faster than training from scratch — Fine-tuning the English GPT-2 in any language with Hugging Face and fastai v2 (practical case with Portuguese). Medium. https://medium.com/@pierre_guillou/faster-than-training-from-scratch-fine-tuning-theenglish-gpt-2-in-any-language-with-hugging-f2ec05c98787

Hair, J. F., Anderson, R. E., Babin, B. J., \& Black, W. C. (2010). Multivariate data analysis: A global perspective (7th ed.). Pearson. https://www.pearson.com/uk/educators/highereducation-educators/program/Hair-Multivariate-Data-Analysis-Global-Edition-7thEdition/PGM916641.html

Haladyna, T. M., \& Rodriguez, M. C. (2013). Developing and validating test items. Routledge. https://www.routledge.com/Developing-and-Validating-Test-Items/HaladynaRodriguez/p/book/9780415876056 
Han, X., Zhang, Z., Ding, N., Gu, Y., Liu, X., Huo, Y., Qiu, J., Yao, Y., Zhang, A., Zhang, L., Han, W., Huang, M., Jin, Q., Lan, Y., Liu, Y., Liu, Z., Lu, Z., Qiu, X., Song, R., . . Zhu, J. (2021). Pre-trained models: Past, present and future. AI Open, 2, 225-250. https://doi.org/10.1016/j.aiopen.2021.08.002

Harrison, P. M. C., Collins, T., \& Müllensiefen, D. (2017). Applying modern psychometric techniques to melodic discrimination testing: Item response theory, computerised adaptive testing, and automatic item generation. Scientific Reports, 7(1). https://doi.org/10.1038/s41598-017-03586-z

Hauser, D. J., Paolacci, G., \& Chandler, J. (2019). Common concerns with MTurk as a participant pool: Evidence and solutions. In F. Kardes, P. Herr, \& N. Schwarz (Eds.), Handbook of research methods in consumer psychology (pp. 319-338). Routledge. http://doi.org/10.4324/9781351137713-17

Hinkin, T. R. (1995). A review of scale development practices in the study of organizations. Journal of Management, 21(5), 967-988. https://doi.org/10.1177/014920639502100509 Ho, A. K., Sidanius, J., Kteily, N., Sheehy-Skeffington, J., Pratto, F., Henkel, K. E., Foels, R., \& Stewart, A. L. (2015). The nature of social dominance orientation: Theorizing and measuring preferences for intergroup inequality using the new $\mathrm{SDO}_{7}$ scale. Journal of Personality and Social Psychology, 109(6), 1003-1028. https://doi.org/10.1037/pspi0000033

Hofstee, W. K., de Raad, B., \& Goldberg, L. R. (1992). Integration of the Big Five and circumplex approaches to trait structure. Journal of Personality and Social Psychology, 63, 146-163. http://dx.doi.org/10.1037/0022-3514.63.1.146

Holland, P. W., \& Wainer, H. (Eds.). (1993). Differential item functioning. https://psycnet.apa.org/record/1993-97193-000 
Holtzman, A., Buys, J., Du, L., Forbes, M., \& Choi, Y. (2020). The curious case of neural text degeneration. ArXiv. https://arxiv.org/pdf/1904.09751.pdf

Hommel, B. E., Wollang, F. J. M., Kotova, V., Zacher, H., \& Schmukle, S. C. (2021). Transformer-based deep neural language modeling for construct-specific automatic item generation. Psychometrika. https://doi.org/10.1007/s11336-021-09823-9

Hopwood, C. J., \& Donnellan, M. B. (2010). How should the internal structure of personality inventories be evaluated? Personality and Social Psychology Review, 14(3), 332-346. https://doi.org/10.1177/1088868310361240

Hornik, K., Stinchcombe, M., \& White, H. (1989). Multilayer feedforward networks are universal approximators. Neural Networks, 2 (5), 359-366. https://doi.org/10.1016/0893-6080(89)90020-8

Howard, J., \& Ruder, S. (2018). Universal language model fine-tuning for text classification. In Proceedings of the 56th Annual Meeting of the Association for Computational Linguistics (Volume 1: Long Papers) (pp. 328-339). https://doi.org/10.18653/v1/P181031

Hu, L., \& Bentler, P. M. (1999). Cutoff criteria for fit indexes in covariance structure analysis: Conventional criteria versus new alternatives. Structural Equation Modeling: A Multidisciplinary Journal, 6(1), 1-55. https://doi.org/10.1080/10705519909540118

Irvine, S. H., \& Kyllonen, P. C. (2002). Item generation for test development. Routledge. https://www.routledge.com/Item-Generation-for-Test-Development/IrvineKyllonen/p/book/9781138973473

Jackson, D. N., \& Messick, S. (1958). Content and style in personality assessment. Psychological Bulletin, 55, 243-252. http://dx.doi.org/10.1037/h0045996 John, O. P. (1989). Towards a taxonomy of personality descriptors. In D. M. Buss \& N. Cantor (Eds.), Personality psychology: Recent trends and emerging directions (pp. 261-271). 
Springer. http://dx.doi.org/10.1007/978-1-4684-0634-4_20

John, O. P. (1990). The "Big Five" factor taxonomy: Dimensions of personality in the natural language and questionnaires. In L. A. Pervin (Ed.), Handbook of personality: Theory and research (pp. 66-100). Guilford Press.

John, O. P., \& Srivastava, S. (1999). The Big Five trait taxonomy: History, measurement, and theoretical perspectives. In L. A. Pervin \& O. P. John (Eds.), Handbook of personality: Theory and research (2nd ed., pp. 102-138). Guilford Press.

Kennedy, B., Ashokkumar, A., Boyd, R. L., \& Dehghani, M. (2021). Text analysis for psychology: Methods, principles, and practices. PsyArXiv. https://doi.org/10.31234/osf.io/h2b8t

Kohno, A., Dahlui, M., Nik Farid, N. D., Aziz, N. A., \& Nakayama, T. (2021). Development of early marriage attitude scale: A multi-dimensional scale for measuring the attitudes toward child marriage. SAGE Open, 11(3), 215824402110376. https://doi.org/10.1177/21582440211037674

Kosh, A. E., Simpson, M. A., Bickel, L., Kellogg, M., \& Sanford-Moore, E. (2019). A costbenefit analysis of automatic item generation. Educational Measurement: Issues and Practice, 38(1), 48-53. https://doi.org/10.1111/emip.12237

Kosinski, M., Matz, S. C., Gosling, S. D., Popov, V., \& Stillwell, D. (2015). Facebook as a research tool for the social sciences: Opportunities, challenges, ethical considerations, and practical guidelines. American Psychologist, 70(6), 543-556. https://doi.org/10.1037/a0039210

Kosinski, M., Wang, Y., Lakkaraju, H., \& Leskovec, J. (2016). Mining big data to extract patterns and predict real-life outcomes. Psychological Methods, 21(4), 493-506. https://doi.org/10.1037/met0000105

Köbis, N., \& Mossink, L. D. (2021). Artificial intelligence versus Maya Angelou: 
Experimental evidence that people cannot differentiate AI-generated from humanwritten poetry. Computers in Human Behavior, 114, 106553. https://doi.org/10.1016/j.chb.2020.106553

Lake, B. M., \& Murphy, G. L. (2021). Word meaning in minds and machines. Psychological Review. https://doi.org/10.1037/rev0000297

Lang, F. R., John, D., Lüdtke, O., Schupp, J., \& Wagner, G. G. (2011). Short assessment of the Big Five: robust across survey methods except telephone interviewing. Behavior Research Methods, 43, 548-567. https://doi.org/10.3758/s13428-011-0066-z

Launay, J., Tommasone, E. L., Pannier, B., Boniface, F., Chatelain, A., Cappelli, A., ... \& Seddah, D. (2021). PAGnol: An extra-large French generative model. arXiv preprint, arXiv:2110.08554.

Lee, J. S., \& Hsiang, J. (2020). Patent claim generation by fine-tuning OpenAI GPT-2. World Patent Information, 62, 101983. https://doi.org/10.1016/j.wpi.2020.101983

Lewis, M., Liu, Y., Goyal, N., Ghazvininejad, M., Mohamed, A., Levy, O., ... \& Zettlemoyer, L. (2019). Bart: Denoising sequence-to-sequence pre-training for natural language generation, translation, and comprehension. arXiv preprint, arXiv:1910.13461.

Lindner, L., Jungo, M., Hennebert, J., Musat, C., \& Fischer, A. (2020). Automatic creation of text corpora for low-resource languages from the internet: The case of Swiss German. ArXiv. https://arxiv.org/abs/1912.00159

Linzen, T., \& Baroni, M. (2021). Syntactic structure from deep learning. Annual Review of Linguistics, 7(1), 195-212. https://doi.org/10.1146/annurev-linguistics-032020-051035

Liu, P., Qiu, X., \& Huang, X. (2016). Recurrent neural network for text classification with multi-task learning. arXiv preprint, arXiv:1605.05101.

Loevinger, J. (1957). Objective tests as instruments of psychological theory. Psychological Reports, 3(3), 635-694. https://doi.org/10.2466/pr0.1957.3.3.635 
Maertens, R., Götz, F. M., Schneider, C. R., Roozenbeek, J., Kerr, J. R., Stieger, S., ... van der Linden, S. (2022). The Misinformation Susceptibility Test (MIST): A psychometrically validated measure of news veracity discernment. PsyArXiv. https://doi.org/10.31234/osf.io/gk68h

Maertens, R., Roozenbeek, J., Basol, M., \& van der Linden, S. (2021). Long-term effectiveness of inoculation against misinformation: Three longitudinal experiments. Journal of Experimental Psychology: Applied, 27(1), 1-16. https://doi.org/10.1037/xap0000315

Manning, C. D., Raghavan, P., \& Schütze, H. (2008). The term vocabulary and postings lists. In Introduction to information retrieval (pp. 44-78). Cambridge University Press.

Marsh, H. W., Lüdtke, O., Muthén, B., Asparouhov, T., Morin, A. J. S., Trautwein, U., \& Nagengast, B. (2010). A new look at the big five factor structure through exploratory structural equation modeling. Psychological Assessment, 22(3), 471-491. https://doi.org/10.1037/a0019227

Marsh, H. W., Morin, A. J., Parker, P. D., \& Kaur, G. (2014). Exploratory structural equation modeling: An integration of the best features of exploratory and confirmatory factor analysis. Annual Review of Clinical Psychology, 10, 85-110. https://doi.org/10.1146/annurev-clinpsy-032813-153700

Marsh, H. W., Muthén, B., Asparouhov, T., Lüdtke, O., Robitzsch, A., Morin, A. J. S., \& Trautwein, U. (2009). Exploratory structural equation modeling, integrating CFA and EFA: Application to students' evaluations of university teaching. Structural Equation Modeling: A Multidisciplinary Journal, 16(3), 439-476. https://doi.org/10.1080/10705510903008220

Marsh, H. W., Nagengast, B., \& Morin, A. J. S. (2013). Measurement invariance of big-five factors over the life span: ESEM tests of gender, age, plasticity, maturity, and la dolce 
vita effects. Developmental Psychology, 49, 1194-1218.

https://doi.org/10.1037/a0026913

Matz, S. C., Appel, R. E., \& Kosinski, M. (2020). Privacy in the age of psychological targeting. Current Opinion in Psychology, 31, 116-121. https://doi.org/10.1016/j.copsyc.2019.08.010

McCrae, R. R., Zonderman, A. B., Costa, P. T., Bond, M. H., \& Paunonen, S. V. (1996). Evaluating replicability of factors in the Revised NEO Personality Inventory: Confirmatory factor analysis versus Procrustes rotation. Journal of Personality and Social Psychology, 70, 552-566. https://doi.org/10.1037/0022-3514.70.3.552

McDonald, R. P. (1999). Test theory: A unified treatment. Psychology Press. https://doi.org/10.4324/9781410601087

Mikolov, T., Karafiát, M., Burget, L., Cernocký, J. H., \& Khudanpur, S. (2010). Recurrent neural network based language model. Proceedings of the 11th International Speech Communication Association, 26-30. https://doi.org/10.21437/Interspeech.2010-343

Mikolov, T., Sutskever, I., Chen, K., Corrado, G. S., \& Dean, J. (2013). Distributed representations of words and phrases and their compositionality. In C. J. C. Burges, L. Bottou, M. Welling, Z. Ghahramani, \& K. Q. Weinberger (Eds.), Advances in Neural Information Processing Systems 26 (pp. 3111-3119). Curran Associates. http://papers.nips.cc/paper/5021-distributed-representations-of-words-and-phrases-andtheir-compositionality.pdf

Millsap, R. E. (2011). Statistical approaches to measurement invariance. Routledge.

Morgado, F. F. R., Meireles, J. F. F., Neves, C. M., Amaral, A. C. S., \& Ferreira, M. E. C. (2017). Scale development: Ten main limitations and recommendations to improve future research practices. Psicologia: Reflexão e Crítica, 30(1). https://doi.org/10.1186/s41155-016-0057-1 
Moshontz, H., Campbell, L., Ebersole, C. R., IJzerman, H., Urry, H. L., Forscher, P. S., Grahe, J. E., McCarthy, R. J., Musser, E. D., Antfolk, J., Castille, C. M., Evans, T. R., Fiedler, S., Flake, J. K., Forero, D. A., Janssen, S. M. J., Keene, J. R., Protzko, J., Aczel, B., . . Chartier, C. R. (2018). The Psychological science accelerator: Advancing psychology through a distributed collaborative network. Advances in Methods and Practices in Psychological Science, 1(4), 501-515. https://doi.org/10.1177/2515245918797607

Nasser, M. A. (2020). Step-by-step guide on how to train GPT-2 on books using Google Colab. Towards Data Science. https://towardsdatascience.com/step-by-step-guide-on-how-totrain-gpt-2-on-books-using-google-colab-b3c6fa15fef0

Nelson, M. J., \& Hoover, A. K. (2020, June). Notes on using Google Colaboratory in AI education. Proceedings of the 2020 ACM Conference on Innovation and Technology in Computer Science Education. https://doi.org/10.1145/3341525.3393997

Nguyen, T. H., Han, H.-R., Kim, M. T., \& Chan, K. S. (2014). An introduction to item response theory for patient-reported outcome measurement. The Patient, 7(1), 23-35. https://doi.org/10.1007/s40271-013-0041-0

Niculescu, M. A., Ruseti, S., \& Dascalu, M. (2021, November). RoGPT2: Romanian GPT2 for text generation. In 2021 IEEE 33rd International Conference on Tools with Artificial Intelligence (ICTAI) (pp. 1154-1161). IEEE.

Nunnally, J. C. (1978). Psychometric Theory (2nd ed.). Mcgraw-Hill College.

Palan, S., \& Schitter, C. (2018). Prolific.ac_-A subject pool for online experiments. Journal of Behavioral and Experimental Finance, 17, 22-27. https://doi.org/10.1016/j.jbef.2017.12.004

Paolacci, G., \& Chandler, J. (2014). Inside the Turk: Understanding Mechanical Turk as a participant pool. Current Directions in Psychological Science, 23(3), 184-188. https://doi.org/10.1177/0963721414531598 
Parker, J. D., Bagby, R., \& Summerfeldt, L. J. (1993). Confirmatory factor analysis of the revised NEO personality inventory. Personality and Individual Differences, 15, 463466. https://doi.org/10.1016/0191-8869(93)90074-d

Paulhus, D. L. (1991). The measurement and control of response bias. In J. P. Robinson, P. R. Shaver, \& L. S. Wrightman (Eds.), Measures of personality and social psychological attitudes (pp. 17-60). Academic Press. http://dx.doi.org/10.1016/B978-0-12-590241$0.50006-X$

Peer, E., Brandimarte, L., Samat, S., \& Acquisti, A. (2017). Beyond the Turk: Alternative platforms for crowdsourcing behavioral research. Journal of Experimental Social Psychology, 70, 153-163. https://doi.org/10.1016/j.jesp.2017.01.006

Perkel, J. M. (2018). By Jupyter, it all makes sense. Nature, 563, 145-146. https://doi.org/10.1038/d41586-018-07196-1

Pituch, K. A., \& Stevens, J. P. (2015). Applied multivariate statistics for the social sciences: Analyses with SAS and IBM's SPSS. Routledge. https://doi.org/10.4324/9781315814919

Pratto, F., Sidanius, J., Stallworth, L. M., \& Malle, B. F. (1994). Social dominance orientation: A personality variable predicting social and political attitudes. Journal of Personality and Social Psychology, 67(4), 741-763. https://doi.org/10.1037/0022-3514.67.4.741

Pugh, D., De Champlain, A., Gierl, M., Lai, H., \& Touchie, C. (2020). Can automated item generation be used to develop high quality MCQs that assess application of knowledge? Research and Practice in Technology Enhanced Learning, 15(1). https://doi.org/10.1186/s41039-020-00134-8

Radford, A., Wu, J., Amodei, D., Clark, J., Brundage, M., \& Sutskever, I. (2019a). Better language models and their implications. OpenAI Blog. https://openai.com/blog/betterlanguage-models/ 
Radford, A., Wu, J., Child, R., Luan, D., Amodei, D., \& Sutskever, I. (2019b). Language models are unsupervised multitask learners. OpenAI Blog. http://www.persagen.com/files/misc/radford2019language.pdf

Raffel, C., Shazeer, N., Roberts, A., Lee, K., Narang, S., Matena, M., ... \& Liu, P. J. (2019). Exploring the limits of transfer learning with a unified text-to-text transformer. arXiv preprint, arXiv:1910.10683.

Rammstedt, B., \& Danner, D. (2017). Acquiescent responding. In V. Zeigler-Hill \& T. K. Shackelford (Eds.), Encyclopedia of personality and individual differences (pp. 1-3). Springer International Publishing. https://doi.org/10.1007/978-3-319-28099-8_1276-1

Rammstedt, B., Danner, D., Soto, C. J., \& John, O. P. (2020). Validation of the short and extrashort forms of the Big Five Inventory-2 (BFI-2) and their German adaptations. European Journal of Psychological Assessment, 36, 149-161. https://doi.org/10.1027/1015-5759/a000481

Rammstedt, B., \& John, O. P. (2007). Measuring personality in one minute or less: A 10-item short version of the Big Five Inventory in English and German. Journal of Research in Personality, 41(1), 203-212. https://doi.org/10.1016/j.jrp.2006.02.001

Rammstedt, B., Lechner, C. M., \& Danner, D. (2021). Short forms do not fall short: A comparison of three (extra-)short forms of the Big Five. European Journal of Psychological Assessment, 37, 23-32. https://doi.org/10.1027/1015-5759/a000574

Rauthmann, J. F. (2020). A (more) behavioural science of personality in the age of multimodal sensing, big data, machine learning, and artificial intelligence. European Journal of Personality, 34(5), 593-598. https://doi.org/10.1002/per.2310

Reise, S. P., Widaman, K. F., \& Pugh, R. H. (1993). Confirmatory factor analysis and item response theory: Two approaches for exploring measurement invariance. Psychological Bulletin, 114(3), 552-566. https://doi.org/10.1037/0033-2909.114.3.552 
Rentfrow, P. J., \& Gosling, S. D. (2003). The do re mi's of everyday life: The structure and personality correlates of music preferences. Journal of Personality and Social Psychology, 84(6), 1236-1256. https://doi.org/10.1037/0022-3514.84.6.1236

Revelle, W., \& Condon, D. M. (2019). Reliability from $\alpha$ to $\omega$ : A tutorial. Psychological Assessment, 31(12), 1395-1411. https://doi.org/10.1037/pas0000754

Risi, S., \& Togelius, J. (2020). Increasing generality in machine learning through procedural content generation. Nature Machine Intelligence, 2(8), 428-436. https://doi.org/10.1038/s42256-020-0208-z

Robins, R. W., Hendin, H. M., \& Trzesniewski, K. H. (2001). Measuring global self-esteem: Construct validation of a single-item measure and the Rosenberg self-esteem scale. Personality \& Social Psychology Bulletin, 27(2), 151-161. https://doi.org/10.1177/0146167201272002

Rosellini, A. J., \& Brown, T. A. (2021). Developing and validating clinical questionnaires. Annual Review of Clinical Psychology, 17(1), 55-81. https://doi.org/10.1146/annurevclinpsy-081219-115343

Royal, K. D., Hedgpeth, M.-W., Jeon, T., \& Colford, C. M. (2019). Automated item generation: The future of medical education assessment? European Medical Journal: Innovations, 2(1), 88-93. https://emj.emg-health.com/wpcontent/uploads/sites/2/2018/01/Automated-Item-Generation.pdf

Schinka, J. A., Velicer, W. F., \& Weiner, I. B. (2012). Handbook of Psychology: Research Methods in Psychology (2nd ed., Vol. 2). Wiley. https://www.wiley.com/en$\mathrm{gb} /$ Handbook+of+Psychology $\% 2 \mathrm{C}+$ Volume $+2 \% 2 \mathrm{C}+$ Research+Methods+in+Psycholo gy\%2C+2nd+Edition-p-9780470890646

Schueler, B. E., Capotosto, L., Bahena, S., McIntyre, J., \& Gehlbach, H. (2014). Measuring parent perceptions of school climate. Psychological Assessment, 26(1), 314-320. 
https://doi.org/10.1037/a0034830

Schumacker, R. E., Lomax, R. G., \& Schumacker, R. (2015). A beginner's guide to structural equation modeling (4th ed.). Routledge. https://www.routledge.com/A-BeginnersGuide-to-Structural-Equation-Modeling-Fourth-Edition/Schumacker-LomaxSchumacker-Lomax/p/book/9781138811935

Sellbom, M., \& Simms, L. J. (2019). Introduction to the special issue on methodological and statistical advancements in clinical assessment. Psychological Assessment, 31(12), 1383-1385. https://doi.org/10.1037/pas0000786

Simms, L. J. (2008). Classical and modern methods of psychological scale construction. Social and Personality Psychology Compass, 2(1), 414-433. https://doi.org/10.1111/j.17519004.2007.00044.x

Slonim, N., Bilu, Y., Alzate, C., Bar-Haim, R., Bogin, B., Bonin, F., Choshen, L., CohenKarlik, E., Dankin, L., Edelstein, L., Ein-Dor, L., Friedman-Melamed, R., Gavron, A., Gera, A., Gleize, M., Gretz, S., Gutfreund, D., Halfon, A., Hershcovich, D., . . . Aharonov, R. (2021). An autonomous debating system. Nature, 591(7850), 379-384. https://doi.org/10.1038/s41586-021-03215-w

Solaiman, I., Clark, J., \& Brundage, M. (2019). GPT-2: 1.5b release. OpenAI Blog. https://openai.com/blog/gpt-2-1-5b-release/

Soto, C. J., \& John, O. P. (2009). Ten facet scales for the Big Five Inventory: Convergence with NEO PI-R facets, self-peer agreement, and discriminant validity. Journal of Research in Personality, 43, 84-90. http://dx.doi.org/10.1016/j.jrp.2008.10.002

Soto, C. J., \& John, O. P. (2017). The next Big Five Inventory (BFI-2): Developing and assessing a hierarchical model with 15 facets to enhance bandwidth, fidelity, and predictive power. Journal of Personality and Social Psychology, 113(1), 117-143. https://doi.org/10.1037/pspp0000096 
Stewart, N., Ungemach, C., Harris, A. J., Bartels, D. M., Newell, B. R., Paolacci, G., \& Chandler, J. (2015). The average laboratory samples a population of 7,300 Amazon Mechanical Turk workers. Judgment and Decision Making, 10(5), 479-491. http://journal.sjdm.org/14/14725/jdm14725.pdf

Sutskever, I., Vinyals, O., \& Le, Q. V. (2014). Sequence to sequence learning with neural networks. Advances in neural information processing systems, 27. https://proceedings.neurips.cc/paper/2014/hash/a14ac55a4f27472c5d894ec1c3c743d2Abstract.html

Tabachnick, B. G., \& Fidell, L. S. (2007). Using multivariate statistics (5th ed.). Pearson. https://psycnet.apa.org/record/2006-03883-000

Topal, M. O., Bas, A., \& van Heerden, I. (2021). Exploring transformers in natural language generation: GPT, BERT, and XLnet. arXiv preprint, arXiv:2102.08036.

Telgarsky, M. (2016). Benefits of depth in neural networks. 29th Annual Conference on Learning Theory, 49, 1517-1539. https://proceedings.mlr.press/v49/telgarsky16.htm van Platen, P. (2020). How to generate text: Using different decoding methods for language generation with Transformers. Hugging Face. https://huggingface.co/blog/how-togenerate

Vassend, O., \& Skrondal, A. (1997). Validation of the NEO Personality Inventory and the fivefactor model. Can findings from exploratory and confirmatory factor analysis be reconciled? European Journal of Personality, 11, 147-166. https://doi.org/10.1002/(SICI)1099-0984(199706)11:2<147::AID-PER278>3.0.CO;2-E

Vaswani, A., Shazeer, N., Parmar, N., Uszkoreit, J., Jones, L., Gomez, A. N., Kaiser, L, \& Polosukhin, I. (2017). Attention is all you need. In I. Guyon, U. V. Luxburg, S. Bengio, H. Wallach, R. Fergus, S. Vishwanathan, \& R. Garnett (Eds.), Advances in neural information processing systems (Vol. 30, pp. 5998-6008). Curran Associates. 
von Chamier, L., Laine, R. F., Jukkala, J., Spahn, C., Krentzel, D., Nehme, E., Lerche, M., Hernández-Pérez, S., Mattila, P. K., Karinou, E., Holden, S., Solak, A. C., Krull, A., Buchholz, T. O., Jones, M. L., Royer, L. A., Leterrier, C., Shechtman, Y., Jug, F., . . . Henriques, R. (2021). Democratising deep learning for microscopy with ZeroCostDL4Mic. Nature Communications, 12(1). https://doi.org/10.1038/s41467-021$22518-0$

von Davier, M. (2018). Automated item generation with recurrent neural networks. Psychometrika, 83(4), 847-857. https://doi.org/10.1007/s11336-018-9608-y von Davier, M. (2019). Training Optimus prime, MD: Generating medical certification items by fine-tuning OpenAI's gpt2 transformer model. arXiv preprint, arXiv:1908.08594. Watson, D. (2004). Stability versus change, dependability versus error: Issues in the assessment of personality over time. Journal of Research in Personality, 38(4), 319350. https://doi.org/10.1016/j.jrp.2004.03.001

Wolf, T., Debut, L., Sanh, V., Chaumond, J., Delangue, C., Moi, A., ... \& Rush, A. M. (2020). Transformers: State-of-the-art natural language processing. In Proceedings of the 2020 Conference on Empirical Methods in Natural Language Processing: System demonstrations (pp. 38-45).

Woolf, M. (2019). How to make custom ai-generated text with GPT-2. Max Woolf's Blog. https://minimaxir.com/2019/09/howto-gpt2/

Worthington, R. L., \& Whittaker, T. A. (2006). Scale development research. The Counseling Psychologist, 34(6), 806-838. https://doi.org/10.1177/0011000006288127

Wu, Y., Schuster, M., Chen, Z., Le, Q. V., Norouzi, M., Macherey, W., ... \& Dean, J. (2016). Google's neural machine translation system: Bridging the gap between human and machine translation. arXiv preprint, arXiv:1609.08144.

Yang, K. (2021). Transformer-based Korean pretrained language models: A survey on three 
years of progress. arXiv preprint, arXiv:2112.03014.

Yang, K. S., \& Bond, M. H. (1990). Exploring implicit personality theories with indigenous or imported constructs: The Chinese case. Journal of Personality and Social Psychology, 58(6), 1087-1095. https://doi.org/10.1037/0022-3514.58.6.1087

Yang, Z., Dai, Z., Yang, Y., Carbonell, J., Salakhutdinov, R. R., \& Le, Q. V. (2019). XLNet: Generalized autoregressive pretraining for language understanding. In $\mathrm{H}$. Wallach, H. Larochelle, A. Beygelzimer, F. d'Alché-Buc, E. Fox, \& R. Garnett (Eds.), Proceedings of the 33rd Conference on Neural Information Processing Systems (NeurIPS 2019) (pp. 5753-5763). Curran Associates.

Zeng, W., Ren, X., Su, T., Wang, H., Liao, Y., Wang, Z., ... \& Tian, Y. (2021). PanGu- $\alpha$ : Large-scale autoregressive pretrained Chinese language models with auto-parallel computation. arXiv preprint, arXiv:2104.12369.

Zhang, Z., Han, X., Zhou, H., Ke, P., Gu, Y., Ye, D., Qin, Y., Su, Y., Ji, H., Guan, J., Qi, F., Wang, X., Zheng, Y., Zeng, G., Cao, H., Chen, S., Li, D., Sun, Z., Liu, Z., . . Sun, M. (2021). CPM: A large-scale generative Chinese pre-trained language model. AI Open, 2, 93-99. https://doi.org/10.1016/j.aiopen.2021.07.001

Zickar, M. J. (2020). Measurement development and evaluation. Annual Review of Organizational Psychology and Organizational Behavior, 7(1), 213-232. https://doi.org/10.1146/annurev-orgpsych-012119-044957

Zielinski, M. J., \& Veilleux, J. C. (2018). The Perceived Invalidation of Emotion Scale (PIES): Development and psychometric properties of a novel measure of current emotion invalidation. Psychological Assessment, 30(11), 1454-1467. https://doi.org/10.1037/pas0000584 